\title{
Restoration of BDNF-TrkB signaling rescues deficits in a mouse model of SCA6
}

Anna A. Cook ${ }^{1}$, Sriram Jayabal ${ }^{1,2,3}$, Jacky Sheng ${ }^{1}$, Eviatar Fields ${ }^{1,2}$, Tsz Chui Sophia Leung ${ }^{1}$, Sabrina Quilez ${ }^{1}$, Eileen McNicholas ${ }^{1}$, Lois Lau ${ }^{1}$, Alanna J. Watt ${ }^{1}{ }^{*}$

${ }^{1}$ Biology Department, McGill University, Montreal, QC, Canada.

${ }^{2}$ Integrated Neuroscience Program, McGill University, Montreal, QC, Canada.

${ }^{3}$ Dept. of Neurobiology, Stanford School of Medicine, Stanford, CA, U.S.A.

*Correspondence: alanna.watt@mcgill.ca 


\section{Abstract (approx. 150 words; currently 151)}

Spinocerebellar ataxia type 6 (SCA6) is a neurodegenerative disease resulting in motor coordination deficits and cerebellar pathology. Expression of brain-derived neurotrophic factor (BDNF) is reduced in several neurodegenerative diseases, including in post-mortem tissue from SCA6 patients. Here, we show that cerebellar BDNF levels are reduced at an early disease stage in a mouse model of SCA6 (SCA6 $\left.{ }^{84 \mathrm{Q} / 84 \mathrm{Q}}\right)$. One month of voluntary exercise was sufficient to elevate BDNF expression, as well as rescue both motor coordination and cerebellar Purkinje cell firing rate deficits. A BDNF mimetic, 7,8- dihydroxyflavone (7,8-DHF) likewise improved motor coordination and reversed Purkinje cell firing rate deficits, suggesting that exercise acts via BDNFTrkB signaling. Prolonged chronic 7,8-DHF administration rescued ataxia when treatment commenced near disease onset, but was ineffective when treatment was started late. These data suggest that 7,8-DHF, which is orally bioavailable and crosses the blood-brain barrier, is a promising therapeutic for SCA6 and argue for the importance of early intervention for SCA6.

Spinocerebellar ataxia type 6 (SCA6) is a rare neurodegenerative disorder characterised by impaired motor coordination and cerebellar pathology. SCA6 is caused by a CAG-repeat expansion mutation in the $C A C N A 1 A$ gene ${ }^{1}$. This leads to a progressive loss of motor coordination, which typically onsets in middle age ${ }^{2}$. There is presently no treatment for $\mathrm{SCA}^{2}$, making an understanding of its pathophysiology and identification of novel treatment strategies a high priority. We have used a mouse model of SCA6 to characterise disease pathophysiology. The

$21 \mathrm{SCA} 6^{84 \mathrm{Q} / 84 \mathrm{Q}}$ model has a CAG-repeat insertion (84 repeats) in the CACNA1A gene ${ }^{3}$. We have previously shown that $\mathrm{SCA} 6^{84 \mathrm{Q} / 84 \mathrm{Q}}$ mice develop motor coordination deficits at $7 \mathrm{months}^{4}$, consistent with the mid-life onset of motor dysfunction in SCA6 patients. Interestingly, Purkinje cell loss occurs long after disease onset in SCA6 ${ }^{84 \mathrm{Q} / 84 \mathrm{Q}}$ mice, at 2 years ${ }^{4}$, reminiscent of Purkinje cell loss observed in post-mortem tissue from SCA6 patients ${ }^{2}$. The long latency between the onset of motor deficits and Purkinje cell loss in mice suggests that early motor coordination deficits in SCA6 patients might arise from altered cerebellar function, rather than from cell death, and thus might be reversible if cerebellar function can be restored.

Neurotrophins are molecules that promote neuronal health and survival in both the developing and 
31 (BDNF) has been observed in post-mortem SCA6 cerebellum ${ }^{6}$, which made us wonder whether BDNF signaling deficits contributed to SCA6 pathology. Indeed, a reduction in BDNF expression is observed in many neurodegenerative diseases, including Huntington's Disease ${ }^{7}$, which like SCA6 is caused by a triplet-repeat expansion mutation, Parkinson Disease (PD) ${ }^{8}$, and Alzheimer Disease $(\mathrm{AD})^{9}$. Additionally, reduced BDNF levels have been reported in several mouse models of ataxia, including SCA1 ${ }^{10,11}$, Stargazer ${ }^{12}$, Lurcher ${ }^{13}$, and Purkinje cell degeneration (pcd) ${ }^{13}$. BDNF signals via two receptors: the high-affinity TrkB receptor, that has been implicated as the disrupted signaling pathway in other disease models ${ }^{14}$, as well as the low-affinity p75 receptor.

To explore whether BDNF signaling deficits contribute to SCA6 pathophysiology, we measured expression levels of both BDNF and its high-affinity receptor, tropomyosin receptor kinase B (TrkB) in SCA6 ${ }^{84 \mathrm{Q} / 84 \mathrm{Q}}$ mice. We found that BDNF and its TrkB receptor were both reduced in the cerebellum at the age of onset of motor coordination deficits. Exercise has been shown to elevate BDNF levels in other brains regions in healthy animals ${ }^{15-18}$ and in mouse models of $\mathrm{AD}^{14,19,20}$. We found that chronic voluntary exercise partially restored cerebellar BDNF levels, as well as rescuing motor coordination deficits. Interestingly, Purkinje cell firing deficits, which we have previously reported in $\mathrm{SCA} 6^{84 \mathrm{Q} / 84 \mathrm{Q}}$ mice $^{21}$, were also reversed, suggesting that Purkinje cell firing deficits can be used as a read-out of cerebellar function in ataxia $^{22}$. To test whether cerebellar BDNF signaling mediated the effects of exercise on ataxia in SCA6, we used a TrkB agonist, 7,8-dihydroxyflavone (7,8-DHF) to mimic BDNF signaling. We found that the BDNF mimetic recapitulated the therapeutic effects of exercise in $\mathrm{SCA} 6^{84 \mathrm{Q} / 84 \mathrm{Q}}$ mice, and that this improvement in cerebellar function was associated with the reversal of Purkinje cell firing rate deficits. Furthermore, since the BDNF mimetic we used is orally bioavailable and crosses the blood brain barrier, its use represents a promising novel therapeutic strategy for SCA6 and other forms of ataxia associated with reduced cerebellar BDNF.

\section{Results}

\section{SCA $6^{84 Q / 84 Q}$ mice display deficits in cerebellar BDNF-TrkB signaling}

Deficits in BDNF signaling have been characterised in multiple neurological diseases ${ }^{7-11,14,19}$. Reduced BDNF levels have been observed in tissue from post-mortem human SCA6 cerebellum ${ }^{6}$, representing an advanced stage of disease progression. We wondered whether BDNF is altered at earlier disease stages, and if altered BDNF levels contribute to disease pathology. To address this 
question, we used a mouse model of SCA6 with a hyper-expanded triplet repeat, SCA6 $6^{84 \mathrm{Q} / 84 \mathrm{Q}}$ mice ${ }^{3}$, which allow us to study early disease stages at the time of onset of motor deficits.

We performed immunohistochemistry for BDNF on sections of cerebellar vermis from littermatched wildtype (WT) and SCA6 ${ }^{84 \mathrm{Q} / 84 \mathrm{Q}}$ mice at 7 months, an age at which we have previously observed the onset of motor deficits ${ }^{4}$. BDNF immunoreactivity was observed in all layers of the cerebellar cortex (Fig. 1a). To determine whether BDNF was expressed in neurons or glia, we costained for calbindin and GFAP to label Purkinje cells and Bergmann glia respectively. We observed a high degree of colocalization between BDNF and calbindin, but little between BDNF and GFAP, indicating that the BDNF signal in the molecular layer reflects localization predominantly in Purkinje cell dendrites (Fig. 1a). We compared BDNF levels in SCA6 $6^{84 \mathrm{Q} / 84 \mathrm{Q}}$ and WT mice and found that BDNF immunoreactivity was significantly reduced in cerebellar slices from SCA6 ${ }^{84 \mathrm{Q} / 84 \mathrm{Q}}$ mice in all three cortical layers (Fig. 1b-d). BDNF is known to be highly expressed in the hippocampus, and BDNF deficits are observed in the hippocampus in both AD and Down syndrome ${ }^{14,19,20}$, so we wondered whether hippocampal BDNF levels were altered in SCA6. We compared BDNF intensity in hippocampal sections from SCA6 ${ }^{84 \mathrm{Q} / 84 \mathrm{Q}}$ and WT mice and observed no significant differences (Supplementary Fig. 1). This suggests that BDNF alterations in $\mathrm{SCA} 6^{84 \mathrm{Q} / 84 \mathrm{Q}}$ mice occur predominantly in the cerebellum, the primary locus of degeneration in $\mathrm{SCA} 6^{23}$.

We investigated the localization and expression levels of the BDNF receptor TrkB by staining cerebellar vermis sections for TrkB, calbindin and GFAP. Similar to BDNF expression, we observed TrkB immunoreactivity in all cerebellar cortical layers, with the strongest signal arising from Purkinje cell bodies, confirmed by colocalization with calbindin (Fig. 1e). TrkB expression in the Purkinje cell and molecular layer colocalized with calbindin but not GFAP (Fig. 1e), suggesting that TrkB is expressed in Purkinje cell dendrites and somata. We observed that TrkB staining was reduced in Purkinje cell somas in $\mathrm{SCA} 6^{84 \mathrm{Q} / 84 \mathrm{Q}}$ mice compared to WT controls, but was not significantly altered in the molecular layer or granule cell layer at disease onset (Fig. 1eh). Thus, we found that both BDNF and its receptor TrkB are reduced in the cerebellar vermis of SCA $6^{84 \mathrm{Q} / 84 \mathrm{Q}}$ mice at 7 months, indicating that a deficit in BDNF signaling is observed in the early stages of disease progression in $\mathrm{SCA} 6^{84 \mathrm{Q} / 84 \mathrm{Q}}$ mice.

\section{Exercise elevates BDNF in the SCA6 ${ }^{84 Q / 84 Q}$ mouse cerebellum}


91 Physical exercise can upregulate BDNF expression in several brain regions ${ }^{15-18}$, including in other 92 neurodegenerative disorders in which BDNF levels are reduced ${ }^{14,19,20}$. We wondered whether exercise could increase BDNF levels in the cerebellum of SCA $6^{84 \mathrm{Q} / 84 \mathrm{Q}}$ mice. To address this, randomly chosen WT and SCA $6^{84 \mathrm{Q} / 84 \mathrm{Q}}$ mice were provided with running wheels (or locked wheels as a sedentary control) for 1 month beginning at 6 months, before the onset of motor deficits has been reported ${ }^{4}$ (Fig. 2a). Both WT and pre-onset SCA6 $6^{84 \mathrm{Q} / 84 \mathrm{Q}}$ mice engaged in running behaviour, although we found that $\mathrm{SCA} 6^{84 \mathrm{Q} / 84 \mathrm{Q}}$ mice ran shorter distances, and with lower intensity. We found no significant differences in the number of active hours each day between WT and SCA $6^{84 \mathrm{Q} / 84 \mathrm{Q}}$ mice (Supplementary Fig. 2).

100

101

102

103

104

105

106

107

108

109

110

111

112

113

114

115

116

To determine whether cerebellar BDNF levels were altered by exercise, we measured BDNF staining intensity in cerebellar vermis of WT and SCA $6^{84 \mathrm{Q} / 84 \mathrm{Q}}$ mice that were either sedentary or exercised (Fig. 2b). We found that exercise elevated BDNF levels in $\mathrm{SCA} 6^{84 \mathrm{Q} / 84 \mathrm{Q}}$ mice in Purkinje cell somas (Fig. 2c; $\mathrm{P}=0.0016$ ), but not in the molecular and granule cell layers (Fig. 2d,e). Interestingly, exercise did not alter BDNF levels in WT mice (Fig. 2c-e), which is consistent with previous reports ${ }^{24-26}$, and argues that chronic exercise increases cerebellar BDNF expression only when it is pathologically reduced, and that levels are likely saturated in WT mice. Thus, although SCA $6^{84 Q / 84 Q}$ mice run less than WT mice when given free access to a running wheel, they exercise at sufficient levels to alter cerebellar BDNF levels.

Since expression of the BDNF receptor TrkB is also reduced in SCA6 $6^{84 Q / 84 Q}$ mice (Fig. 1f), and because TrkB expression is itself influenced by its ligand $\mathrm{BDNF}^{27}$, we wondered whether its expression was also modulated by exercise. We found that TrkB levels were not significantly different in exercised or sedentary SCA6 ${ }^{84 \mathrm{Q} / 84 \mathrm{Q}}$ mice (Fig. 2g-j; Purkinje cell layer $\mathrm{P}=0.14$, molecular layer $\mathrm{P}>0.99$, granule cell layer $\mathrm{P}>0.99$ ), although we saw a modest reduction in TrkB receptor expression in Purkinje cell soma in WT mice (Fig. $\mathbf{2 g}$; $\mathrm{P}=0.016)$. This suggests that in SCA $6^{84 \mathrm{Q} / 84 \mathrm{Q}}$ mice, exercise enhances cerebellar BDNF expression without affecting expression of its high-affinity TrkB receptor.

\section{Exercise alleviates motor coordination and Purkinje cell firing deficits in SCA6 ${ }^{84 Q / 84 Q}$ mice}

Exercise has been shown to rescue deficits in an SCA1 mouse model ${ }^{28,29}$ and in ataxic Snf2h-null mice $^{30}$, although BDNF has not been identified as the mechanism underlying its therapeutic action 
in these models. We wondered whether exercise, perhaps via elevation of cerebellar BDNF levels, would rescue motor behavioural deficits in $\mathrm{SCA} 6^{84 \mathrm{Q} / 84 \mathrm{Q}}$ mice. To test this, we used an accelerating rotarod to assay motor coordination (Fig. 3a). We found that motor coordination in $\mathrm{SCA} 6^{84 \mathrm{Q} / 84 \mathrm{Q}}$ mice was significantly improved after 1 month of exercise, compared to sedentary SCA6 $6^{84 \mathrm{Q} / 84 \mathrm{Q}}$ mice (Fig. 3b), although not to WT levels (Fig. 3b). Interestingly, consistent with the lack of change in BDNF expression levels, WT mice showed no significant changes with exercise compared to sedentary controls (Fig. 3b). This shows that in addition to restoring cerebellar BDNF levels, exercise reduces ataxia in $\mathrm{SCA} 6^{84 \mathrm{Q} / 84 \mathrm{Q}}$ mice.

We next wondered whether other cellular deficits in SCA $6^{84 \mathrm{Q} / 84 \mathrm{Q}}$ mice were altered by exercise. We have previously shown that spontaneous Purkinje cell firing frequency and regularity are reduced at 7 months in $\mathrm{SCA} 6^{84 \mathrm{Q} / 84 \mathrm{Q}}$ mice $^{21}$. Similar deficits in intrinsic Purkinje cell firing have been observed in several ataxia models ${ }^{10,21,31-33}$ (summarised $i^{22}$ ), suggesting that such firing deficits may be a read-out of cerebellar dysfunction in ataxia. Using juxtacellular recordings of spontaneous Purkinje cell firing in acute sagittal slices, we found that exercise rescued Purkinje cell firing frequency deficits in $\mathrm{SCA} 6^{84 \mathrm{Q} / 84 \mathrm{Q}}$ mice to levels indistinguishable from WT mice (Fig. 3c). However, firing regularity, quantified by the coefficient of variation (CV) of inter-spike intervals, was unaltered and remained significantly higher in exercise SCA $6^{84 \mathrm{Q} / 84 \mathrm{Q}}$ mice compared to WT controls (Fig. 3d). Exercise did not significantly alter firing properties of Purkinje cells in WT mice (Fig. 3d), consistent with the finding that BDNF levels and motor coordination are unaltered by exercise in WT mice. Thus, exercise restores Purkinje cell firing rate and motor coordination deficits, perhaps by restoring cerebellar BDNF levels.

The BDNF mimetic 7,8-DHF improves motor coordination and Purkinje cell firing rate deficits in $\mathrm{SCA} 6^{84 Q / 84 Q}$ mice

If the behavioural rescue we observed in pre-onset mice with exercise was due to enhanced BDNF expression, we wondered whether targeting BDNF-TrkB signaling alone would be sufficient to rescue motor coordination. To test this, we administered a small molecule TrkB agonist, 7,8dihydroxyflavone ${ }^{14,34}(7,8-\mathrm{DHF})$ in drinking water (Fig. 4a, b).

SCA $6^{84 \mathrm{Q} / 84 \mathrm{Q}}$ mice were given access to drinking water with sucrose and either 7,8 -DHF or DMSO vehicle alone as a control at 6 months, prior to onset of motor deficits (Fig. 4b), after which we 
tested motor coordination using the rotarod assay. We found that after 1 month, 7,8-DHF administration alleviates motor coordination deficits in SCA6 ${ }^{84 \mathrm{Q} / 84 \mathrm{Q}}$ mice compared to vehicle controls (Fig. 4c). Furthermore, we observed a weak positive correlation between the amount of 7,8-DHF consumed daily and rotarod performance (Supplementary Fig. 3), supporting the hypothesis that 7,8-DHF intake influences motor coordination. If 7,8-DHF acts in the cerebellum of $\mathrm{SCA} 6^{84 \mathrm{Q} / 84 \mathrm{Q}}$ mice to improve motor coordination, we would predict that it would restore Purkinje cell firing abnormalities ${ }^{22}$. To test this, we carried out juxtacellular recordings of intrinsic Purkinje cell action potentials, and found that similar to the effects of exercise, 7,8-DHF elevated Purkinje cell firing frequency but not firing regularity deficits in SCA6 $6^{84 \mathrm{Q} / 84 \mathrm{Q}}$ mice (Fig. 4d, e). Thus chronic 7,8-DHF treatment recapitulated the therapeutic effect of the exercise on motor coordination, and also reverses Purkinje cell firing rate deficits that have been previously associated with impaired motor coordination ${ }^{21,22}$. These findings support our hypothesis that exercise and 7,8-DHF act via the same signaling pathway in $\mathrm{SCA} 6^{84 \mathrm{Q} / 84 \mathrm{Q}}$ mice.

We hypothesize that WT BDNF levels are near saturation since exercise does not influence motor coordination (Fig. 3b), BDNF levels (Fig. 2d-f), or Purkinje cell firing properties (Fig. 3d) in these mice. We thus expected that 7,8-DHF would not have an effect on motor coordination in WT mice. To test this, we administered 7,8-DHF to WT mice for 1 month, and found it did not significantly affect motor coordination (Supplementary Fig. 4). This reinforces our hypothesis that 7,8-DHF affects motor coordination only when cerebellar BDNF levels are reduced.

If exercise restores motor coordination in $\mathrm{SCA} 6^{84 \mathrm{Q} / 84 \mathrm{Q}}$ mice through elevation of cerebellar BDNF as we hypothesize, we predict that the combination of exercise and 7,8-DHF treatments should not be additive, whereas if they restore motor coordination through different pathways, combining these treatments would be additive. To differentiate between these possibilities, we compared the effects on ataxia provided by exercise, 7,8-DHF, and the combination of these (Fig. 5a, b). We found that exercise alone, exercise $+7,8$-DHF and 7,8-DHF alone all significantly rescued motor coordination in SCA6 $6^{84 \mathrm{Q} / 84 \mathrm{Q}}$ mice compared to sedentary SCA6 $6^{84 \mathrm{Q} / 84 \mathrm{Q}}$ mice, but that there was no significant difference in performance between these three conditions (Fig. 5c). Notably, mice administered Exercise + 7,8-DHF demonstrated no additional improvement in motor coordination in in $\mathrm{SCA} 6^{84 \mathrm{Q} / 84 \mathrm{Q}}$ mice compared to either treatment alone (Fig. 5b). This supports the hypothesis that exercise improves motor coordination in $\mathrm{SCA} 6^{84 \mathrm{Q} / 84 \mathrm{Q}}$ mice by enhancing $\mathrm{BDNF}-\mathrm{TrkB}$ 
signaling in the cerebellum. These data suggest that both exercise and 7,8-DHF improve motor coordination via TrkB signaling, and that signaling is near saturation in both conditions.

\section{BDNF mimetic 7,8-DHF can restore motor coordination at later disease stages when administration starts early}

SCA6 is a progressive disorder which means that motor coordination worsens over time in both patients and mouse models ${ }^{2,4}$. To determine whether targeting TrkB signaling at later ages in SCA $6^{84 \mathrm{Q} / 84 \mathrm{Q}}$ mice would improve motor coordination, we administered 7,8-DHF at 7 months, after disease onset. SCA6 $6^{84 \mathrm{Q} / 84 \mathrm{Q}}$ mice were assayed on rotarod for 5 days and then divided into two equally performing groups, which were given either 7,8-DHF or DMSO vehicle in drinking water (Fig. 6a). Rotarod testing continued daily to determine if and when changes in motor coordination occurred. SCA6 $6^{84 \mathrm{Q} / 84 \mathrm{Q}}$ mice treated with 7,8 -DHF performed significantly better than untreated controls after 14 days, and performance tended to increase for the next 2 weeks in treated mice (Fig. 6b). The relative slow time-course of 7,8-DHF action is consistent with our finding that 4hour 7,8-DHF treatment on acute cerebellar slices was insufficient to alter Purkinje cell firing properties (Supplementary Fig. 6), supporting our observation of a slow time course to ameliorate motor coordination (Fig. 6b). Our findings that restoring TrkB signaling with 7,8-DHF at the onset of ataxia still rescued motor coordination in $\mathrm{SCA} 6^{84 \mathrm{Q} / 84 \mathrm{Q}}$ mice are particularly promising since mice did not exercise at high levels at this age, and exercise was unable to rescue deficits in $\mathrm{SCA} 6^{84 \mathrm{Q} / 84 \mathrm{Q}}$ mice when started after onset, even when supplemented with bouts of forced exercise on a treadmill (Supplementary Fig. 5). The difference we observe in the efficacy of exercise and 7,8-DHF treatment post-onset may arise from motor impairment due to disease progression that prevents $\mathrm{SCA} 6^{84 \mathrm{Q} / 84 \mathrm{Q}}$ mice from exercising at sufficiently high levels.

Given that BDNF expression is regulated by BDNF-TrkB signaling ${ }^{27}$ we wondered if the effects of 7,8-DHF might persist in SCA6 ${ }^{84 \mathrm{Q} / 84 \mathrm{Q}}$ mice. To test this, we withdrew 7,8-DHF after 1 month while testing motor coordination weekly. We found that 7,8-DHF-treated mice maintained elevated motor coordination for 2 weeks after 7,8-DHF was removed, but that motor coordination returned to control levels by 3 weeks (Fig. 6c). Thus, 7,8-DHF treatment had an effect over a slow time course on motor coordination both for drug administration and withdrawal. 
SCA6 patients often live with the progressively worsening disease for decades. We wondered whether 7,8-DHF could provide long-lasting benefits in SCA $6^{84 \mathrm{Q} / 84 \mathrm{Q}}$ mice. To address this, we continued 7,8-DHF treatment for 4 months in a subset of SCA $6^{84 \mathrm{Q} / 84 \mathrm{Q}}$ mice and assayed motor coordination monthly (Fig. 7a). We found that SCA6 $6^{84 \mathrm{Q} / 84 \mathrm{Q}}$ mice chronically administered 7,8DHF showed improved performance at 7, 9, and 10 months (Fig. 7b). This suggests that the continued administration of 7,8-DHF may have a long-lasting therapeutic benefit in SCA6 patients. To determine whether chronic 7,8-DHF administration had similar cellular effects in older mice that it did in younger mice, we recorded Purkinje cell firing rates in acute slices made after 4 months of 7,8-DHF and found that they were not significantly different than controls (Fig. 7c), unlike what we observed in younger mice where 7,8-DHF restored Purkinje cell firing rate deficits (Fig. 4c).

Our observation that treatment with the BDNF mimetic 7,8-DHF provides long-term therapeutic benefits in the SCA6 ${ }^{84 \mathrm{Q} / 84 \mathrm{Q}}$ mouse model (Fig. 7b) is promising for SCA6 patients. However, SCA6 is often diagnosed after disease onset, meaning that early treatment may not always be practical. We thus wondered whether 7,8-DHF would ameliorate ataxia in SCA6 ${ }^{84 \mathrm{Q} / 84 \mathrm{Q}}$ mice when drug administration was initiated at a later stage of disease progression. To determine whether this was likely to succeed, we first determined that TrkB receptors were still expressed in 9 month SCA6 ${ }^{84 \mathrm{Q} / 84 \mathrm{Q}}$ cerebellum (Supplementary Fig. 7), suggesting that the receptor target for 7,8-DHF is expressed in the SCA6 brain. We then administered 7,8-DHF for 1 months to SCA6 $6^{84 \mathrm{Q} / 84 \mathrm{Q}}$ mice at 9 months (Fig. 7d). We observed no significant improvement in motor coordination in latetreated 7,8-DHF mice (Fig. 7e), and similarly, observed no changes in Purkinje cell firing properties (Fig. 7f). These data suggest that the lack of a therapeutic effect of late-administered 7,8-DHF is unlikely to arise due to TrkB receptors, but rather likely arises from changes in downstream signaling mechanisms. Taken together, our results demonstrate that $7,8-\mathrm{DHF}$ is a promising therapeutic with the potential for long-lasting benefits for SCA6-associated ataxia when administration is started around disease onset, but not at later stages.

\section{Discussion}

We identified BDNF-TrkB signaling disruptions as a contributor to pathophysiology at early disease stages in SCA6 ${ }^{84 \mathrm{Q} / 84 \mathrm{Q}}$ mice and successfully targeted this pathway to improve disease phenotype using two approaches. Our work represents a promising novel therapeutic strategy for 
SCA6 and sheds light on the mechanisms underlying the therapeutic properties of exercise for SCA6. Reduced cerebellar BDNF has been reported in post mortem brain tissue from SCA6 patients ${ }^{6}$ and we identified similar changes in the SCA $6^{84 Q / 84 Q}$ mouse model, alongside reduced levels of the BDNF receptor TrkB. We went on to show that cerebellar BDNF could be rescued by a chronic exercise program, which also rescued deficits in motor coordination and Purkinje cell firing. We then used 7,8-DHF, a BDNF mimetic that acts as a TrkB agonist, to rescue deficits in the SCA $6^{84 \mathrm{Q} / 84 \mathrm{Q}}$ mouse. Combining these two therapies provided no additional rescue of motor coordination deficits, suggesting that exercise exerts its beneficial effects on motor coordination by restoring TrkB signaling in the cerebellum. 7,8-DHF treatment improved motor coordination for months if treatment was initiated around the onset of ataxia, but was insufficient if treatment was started later in the disease progression.

Many studies using animal models of human diseases focus on early, peri-onset disease stages (e.g. $\left.{ }^{21}\right)$. Yet for progressive, neurodegenerative diseases like SCA6, patients often live with symptoms for years or decades, and diagnosis is sometimes made long after disease onset. For this reason, we chose to focus on multiple stages of disease progression: pre-onset, early progression, and late progression. We found that in our mouse model, both exercise and TrkB agonist treatment

253 mitigated the development of motor coordination and Purkinje cell firing deficits when 254 administered pre-onset. Exercise did not contribute further improvement of motor coordination by 7,8-DHF suggesting that exercise and 7,8-DHF act via the same pathway. Later, as disease 256 progressed, exercise ceased to rescue motor function, likely because the mice did not run far enough. Nonetheless, treatment with 7,8-DHF was still able to reverse deficits, demonstrating that restoring BDNF was still possible at early stages of disease progression. This argues that different windows of opportunity may exist for different interventions, although exercise in humans after disease onset may be more successful than it was in our mouse model. Importantly, however, at a more advanced stage later in disease progression, 7,8-DHF treatment was only able to restore 262 motor when administration began peri-onset and continued for 4 months, but not when 263 administration began later. With increased prevalence of predictive genetic testing in 264 neurodegenerative diseases like $\mathrm{SCA}^{35}$, an awareness of the importance of early treatment may 265 be of benefit to patients. 
266 Exercise is an intervention that is accessible to many patients and has been successfully used to improve outcomes for ataxia; for example, exercise leads to some alleviation of motor symptoms and prevention of cerebellar decline in patients with various types of ataxia, including SCA6 ${ }^{36-39}$, although until now there was no evidence that this therapeutic action was mediated by BDNF. Exercise has also proven therapeutic in other animal models of ataxia ${ }^{28-30}$, although the mechanism of rescue described in those models appears at least partially different from the mechanism we describe here, perhaps because of differences in the duration and intensity of exercise animals undergo. It is also likely that the difference in the molecular mechanism of therapeutic exercise in different ataxias arises due to differences in the molecular pathophysiology of different disorders. While it is unclear what the equivalent of 30 days of voluntary wheel running by a mouse would be for an SCA6 patient, we know that there are exercise protocols, such as high-intensity interval training, that target BDNF-TrkB signaling in people ${ }^{40,41}$. Thus, despite our findings that exercise ameliorates ataxia may be generalizable, it will be nonetheless important to determine how exercise acts in each form of ataxia, to determine whether a strategy targeting BDNF signaling will be beneficial.

While the upregulation of BDNF with exercise has been well characterised in the hippocampus ${ }^{42}$, where even short bouts of exercise lead to upregulation of BDNF, the picture in the cerebellum has been less clear. Some studies in rodents have reported no change in BDNF expression in the cerebellum with exercise ${ }^{24-26}$, while others report that exercise increases levels of cerebellar BDNF $^{15,43}$. These discrepancies could arise due to differences in the duration and type of exercise in each protocol. We found that although 4 weeks of exercise did not alter cerebellar BDNF protein levels in WT mice, it did increase BDNF protein levels in the SCA6 $6^{84 Q / 84 Q}$ cerebellum. This differential effect on WT and disease model mice is not unprecedented, and other studies similarly report that exercise acts differently in different models and circumstances ${ }^{44,45}$. We surmise that the chronic exercise protocol that we used increased BDNF levels only when they were already pathologically reduced in the SCA $6^{84 \mathrm{Q} / 84 \mathrm{Q}}$ mice.

Reduced BDNF expression is a hallmark of many neurological diseases. BDNF is known to be upregulated by neuronal activity, and so the reduction in SCA6 and other ataxias could be a secondary effect caused by the decreased firing frequency of Purkinje cells that has been reported for $\mathrm{SCA}^{21}$ and many other ataxic mouse models ${ }^{22}$. However, other mechanisms leading to 
reduced BDNF in disease cannot be excluded, and indeed, BDNF disruption may arise from a variety of different insults in different diseases.

The restoration of BDNF-TrkB signaling we describe here for SCA6 is a promising potential therapeutic approach for multiple ataxias. Our results are consistent with previous findings that viral reintroduction of the BDNF gene rescued ataxia in Stargazer mice ${ }^{12}$ and acute cerebellar delivery of BDNF to SCA1 $A T X N 1(82 Q)$ mouse model had a similar therapeutic effect ${ }^{11,46}$. However, overexpression of BDNF has been associated with negative outcomes in some cases ${ }^{47-}$ ${ }^{49}$, likely due to the complex signaling pathways that are known to be activated by BDNF. Thus, instead of virally reintroducing BDNF in the SCA6 $6^{84 \mathrm{Q} / 84 \mathrm{Q}}$ mouse, we chose to use a different approach administering a BDNF mimetic that acts as a TrkB agonist, as this signals exclusively through the neuroprotective TrkB pathway and may be more translatable to the clinic ${ }^{5}$.

In summary, we have found that both BDNF and its high-affinity TrkB receptor are downregulated at the age of onset in a mouse model of SCA6. We found that exercise elevated BDNF in the cerebellum of SCA6 mice, and that this was associated with an improvement of motor coordination deficits and cellular firing deficits in cerebellar Purkinje cells. We then used a BDNF mimetic to activate TrkB receptors directly and found this restored motor coordination and Purkinje cell firing deficits in a manner similar to exercise. When administered together, exercise and the BDNF mimetic were not additive, suggesting that they were acting on the same pathway. Chronic administration of the BDNF mimetic that was initiated around the age of disease onset led to a sustained improvement in motor coordination, but this was not true when administration started later during disease progression. Taken together, our data suggest that exercise modulates ataxia in SCA6 through the restoration of BDNF-TrkB signaling in the cerebellum and that a BDNF mimetic can mimic this therapeutically. Our work therefore highlights a promising therapeutic approach to both SCA6 and other neurodegenerative diseases.

\section{Methods}

\section{Animals}

We used a knock-in mouse model of SCA6 with a humanised 84 CAG repeat expansion mutation at the $C A C N A 1 A$ locus $\left(\mathrm{SCA}^{84 \mathrm{Q}}\right)$. Mice that were heterozygous at this locus were obtained from Jackson laboratories (Bar Harbor, Maine; strain: B6.129S7-Cacnalatm3Hzo/J; stock number: 
008683) and were bred to obtain SCA6 $6^{84 \mathrm{Q} / 84 \mathrm{Q}}$ and litter-matched WT control mice. Genotyping was performed at weaning using primer sequences published by Jackson laboratories. Male and female mice were used in all experiments. All animal procedures were carried out with the approval of the McGill Animal Care Committee in accordance with the Canadian Council on Animal Care guidelines.

\section{Immunohistochemistry}

Tissue fixation was carried out as previously described ${ }^{31}$. Mice were deeply anesthetised with intraperitoneal injection of 2,2,2-tribromoethanol (Avertin) and an intracardiac perfusion was performed with a flush of ice-cold phosphate-buffered saline (PBS, 0.1M, pH 7.4) with $5.6 \mu \mathrm{g} / \mathrm{mL}$ heparin salt, followed by $40 \mathrm{~mL}$ of $4 \%$ paraformaldehyde (PFA) in phosphate buffer (PB, pH 7.4). Extracted tissue was stored in $4 \% \mathrm{PFA}$ at $4^{\circ} \mathrm{C}$ for a further 24 hours of fixation before being transferred to PBS with $0.5 \%$ sodium azide for storage at $4{ }^{\circ} \mathrm{C}$. The cerebellar vermis was dissected and sliced into $100 \mu \mathrm{m}$ sagittal slices with a Vibratome 3000 sectioning system (Concord, ON, Canada). Immunohistochemistry on free floating slices was carried out with antibodies for proteins of interest along with anti-calbindin or anti-GFAP as markers for Purkinje cells and Bergmann glia, respectively. Antibodies are listed in Supplementary Table 1. The use of anti-BDNF included an additional heat-induced epitope retrieval step comprising 10 minutes of heating to $95^{\circ} \mathrm{C}$ in PBS, after which slices were allowed to cool before proceeding with the primary antibody staining. After staining, slices were immediately mounted in ProLong Gold Antifade mounting medium (ThermoFisher Scientific, Waltham, USA) and stored in the dark at $4^{\circ} \mathrm{C}$.

\section{Image acquisition and analysis}

Imaging was performed using an LSM800 confocal microscope (Zeiss), using Zeiss Zen software for image acquisition. The raw fluorescence intensity was recorded in arbitrary units and was normalised to the mean WT value within a batch. Due to limitations of litter size, immunocytochemistry was typically carried out in several batches. To minimise batch-specific effects on the data, all data is shown normalised to the mean WT value of that batch (defining WT intensity = 1). Image analysis was performed in FIJI (ImageJ; US National Institutes of Health $)^{50,51}$. Raw images of lobule 3 of cerebellar vermis were used and manual tracing around Purkinje cell bodies in the calbindin channel allowed the delineation of ROIs around the Purkinje cell bodies. Missing or out of the plane of focus cells were skipped. These ROIs were then exported 
onto the BDNF/TrkB channel and fluorescence intensity was measured (integrated density, arbitrary units). For measurements of fluorescence intensity in the molecular layer and granule cell layer, we used 4 ROIs of consistent size $\left(5193 \mu \mathrm{m}^{2}\right)$ to determine the total intensity at randomly chosen locations in the molecular and granule cell layers (Supplementary Fig. 8).

\section{Voluntary running}

Mice were individually housed in low profile rat cages with running wheels (Columbus Instruments, $\mathrm{OH}, \mathrm{USA})$. Sedentary control mice received wheels that were locked in position to control for environmental enrichment provided by the presence of the wheel and the larger cages. The number of wheel rotations was recorded hourly using Multi-Device software (Columbus Instruments, $\mathrm{OH}, \mathrm{USA})$.

\section{Treadmill exercise}

Mice were moved to the behavior room for acclimatization one hour prior to the start of exercise. Mice were placed individually onto an enclosed single lane treadmill (Treadmill Simplex II Columbus Instruments). The treadmill speed was set to $6 \mathrm{~m} / \mathrm{min}$ for the first 5 minutes, then increased to $10 \mathrm{~m} / \mathrm{min}$ for 5 minutes, then increased in $1 \mathrm{~m} / \mathrm{min}$ increments for each subsequent 5 minute period of the 20-minute exercise period. The treadmill was equipped with a shock plate, which distributed $2 \mathrm{~mA}$ shocks twice per minute when mice stepped off the treadmill. If a mouse remained on the shock plate for more than 30 seconds, the treadmill was stopped for an additional 30 seconds allowing the mouse to return to the treadmill. Mice in the control condition were placed on the stationary treadmill and allowed to explore it for 20 minutes with the shock platform on, encouraging the mice to remain on the treadmill. This exercise protocol was repeated daily for 6 weeks.

\section{Accelerating rotarod}

We used an accelerating protocol on a Rotarod (Stoelting, IITC) as previously described ${ }^{4,21,31}$. We have previously shown that this assay is able to detect differences in motor coordination in WT and $\mathrm{SCA} 6^{84 \mathrm{Q} / 84 \mathrm{Q}}$ mice in both control and treatment conditions ${ }^{4,21}$. Mice were moved to the testing room and left to acclimatise for 1 hour prior to testing. Mice underwent 4 trials per day with a 1015 minute rest period between trials. Testing was carried out at the same time each day for 5 consecutive days or in some cases for up to 30 days. The protocol started with a speed of 4 RPM 
and then accelerated to 40 RPM over 5 minutes after which the assay continued at 40 RPM for up to 5 further minutes. Latency to fall off the rotarod was recorded in each trial.

\section{Acute Slice preparation}

Slice preparation was carried out as previously described ${ }^{21}$. Mice were deeply anesthetised with intraperitoneal injection of 2,2,2-tribromoethanol (Avertin) and an intracardiac perfusion was performed with ice cold partial sucrose replacement slicing solution $(111 \mathrm{mM}$ Sucrose, $50 \mathrm{mM}$ $\mathrm{NaCl}, 2.5 \mathrm{mM} \mathrm{KCl}, 0.65 \mathrm{mM} \mathrm{CaCl} 2,10 \mathrm{mM} \mathrm{MgCl} 2,1.25 \mathrm{mM} \mathrm{NaH} 2 \mathrm{PO} 4,25 \mathrm{mM}$ NaHCO3 and $25 \mathrm{mM}$ glucose, bubbled with $95 \% \mathrm{O} 2$ and 5\% CO2 to maintain $\mathrm{pH}$ at 7.3 ; osmolality $\sim 320$ mOsm). The cerebellar vermis was dissected and sliced into $200 \mu \mathrm{m}$ sagittal slices with a VT 1200S vibratome (Leica Microsystems, Wetzlar, Germany). Slices were incubated in artificial cerebrospinal fluid (ACSF: $125 \mathrm{mM} \mathrm{NaCl}, 2.5 \mathrm{mM} \mathrm{KCl}, 2 \mathrm{mM} \mathrm{CaCl} 2,1 \mathrm{mM} \mathrm{MgCl} 2,1.25 \mathrm{mM}$ $\mathrm{NaH} 2 \mathrm{PO} 4,26 \mathrm{mM} \mathrm{NaHCO} 3$ and 20mM glucose, bubbled with 95\% O2 and 5\% CO2 to maintain $\mathrm{pH}$ at 7.3 ; osmolality $\sim 320 \mathrm{mOsm}$ ) bubbled with carbogen at $37^{\circ} \mathrm{C}$ for 45 minutes and then transferred to room temperature for the duration of experiments. All reagents were obtained from Sigma (Oakville, ON, Canada) apart from $\mathrm{MgCl}_{2}$ and $\mathrm{CaCl}_{2}$ obtained from Fisher Scientific (Toronto, ON, Canada).

\section{Electrophysiology}

Recordings of spontaneous Purkinje cell firing in lobule 3 of cerebellar vermis were carried out as previously described ${ }^{21,31}$. Slices were kept in a bath of ACSF at approximately $33^{\circ} \mathrm{C}$ during recording. Purkinje cells were visually identified and juxtacellular recordings were made at the soma with borosilicate pipettes pulled with a P-1000 puller with tip sizes of 1-2 $\mu \mathrm{m}$ (Sutter Instruments, Novato, CA, USA). Data acquisition and analysis was performed in IGOR Pro version 6.0 and 7.0 (Wavemetrics, Portland, OR, USA) using custom routines.

\section{7,8-DHF administration}

7,8-dihydroxyflavone (7,8-DHF) is orally bioavailable and previous studies have demonstrated that oral administration via drinking water is both well tolerated and able to deliver therapeutically relevant dosages of the drug in mice ${ }^{14}$. Single-housed mice were given free access to solutions containing either 157.3 $\mu \mathrm{M}$ 7,8-DHF (TCI America) dissolved in 0.04\% DMSO or control solutions with $0.04 \%$ DMSO only, dissolved in autoclaved water. $10 \%$ sucrose was added to both 
413 solutions to make them palatable, as previously described ${ }^{21}$. Solutions were made fresh twice

414 weekly and refreshed daily, with solutions stored at $4^{\circ} \mathrm{C}$ in between refills. Consumption was

415 monitored daily to determine the administered dose of 7,8-DHF. Mice consumed on average 21

$416 \mathrm{~mL}$ of the sucrose-sweetened drug solution daily, giving a daily dose of $0.84 \mathrm{mg} 7,8$-DHF. The

417 control group with $0.04 \%$ DMSO and 10\% sucrose but no 7,8-DHF consumed on average $19 \mathrm{~mL}$

418 of the solution each day. The drug treatment period ranged from 25 days to 4 months and mice

419 underwent behavioural testing on the rotarod assay during this time. Mice were monitored daily

420 when solutions were administered and no adverse health effects were observed. Mice were

421 weighed at least every second day during behavioural testing, meaning that some mice receiving

422 7,8-DHF were weighed throughout their drug treatment and did not gain or lose weight during the

423 treatment (Supplementary Fig. 3). Reagents were obtained from Sigma (Oakville, ON, Canada)

424 unless otherwise specified.

\section{Statistics}

426 Comparisons were made using either one-way ANOVA followed by post hoc tests using JMP 427 software (SAS, Cary, NC), or two-tailed Student's $t$ tests or Mann-Whitney $U$ tests using Igor Pro 428 software, for parametric or non-parametric testing respectively. Data are reported as 429 Mean \pm S.E.M. when normally distributed, or as median when the data are not normally 430 distributed.

\section{Author Contributions}

432 A.A.C designed and ran experiments and analyzed data for all Figures except Fig. 3 and wrote the manuscript, S.J. designed and ran experiments and analyzed data for Fig. 3 and helped write the manuscript, J.S. designed and ran experiments and analyzed data for Fig. 1 and 2, E.F. ran 435 experiments for Fig. 7 and analyzed data for Fig. 2, T.C.S.L. analyzed data for Supplementary Fig.

436 7, S.Q. ran experiments and analyzed data for Fig. 3, E. M. ran experiments and analyzed data for 437 Supplementary Fig. 4, L.L. analyzed data for Supplementary Fig. 1, 2 and 3, A.J.W. conceived of 438 the project, designed experiments and analyzed data, supervised the project, and wrote the 439 manuscript. 
441 We thank Anne McKinney, Aparna Suvrathan and Jesper Sjöström for helpful advice and 442 thoughtful feedback on the project. Custom Igor software routines for electrophysiology data

443 acquisition and analysis were written by Jesper Sjöström. We thank Karim Nader and Arkady 444 Khoutorsky for the use of equipment. We thank all current and former members of the Watt lab 445 for their input, particularly Kristen Vieira-Lomasney, Mohini Bhade and Genavieve Maloney who 446 provided technical support, and Brenda Toscano-Márquez, Amy Smith-Dijak, Daneck Lang447 Ouellette, Kim Gruver, and Louisa Shen for their helpful feedback. Imaging was performed in the 448 McGill University Advanced BioImaging Facility (ABIF) and we thank ABIF staff members for 449 their technical support. We are grateful for the excellent animal care and training we received from 450 the McGill Animal Resources Centre (CMARC), particularly Tanya Koch and Juan Canale who 451 greatly facilitated the research.

452 Financial Support

453 This work was funded by a McGill Biology Doctoral Excellence Award (AAC), and Graduate 454 Student Fellowships from the Canada First Research Excellence Fund awarded to McGill 455 University for the Healthy Brains for Healthy Lives initiative (AAC, EF), two returning student 456 awards from the McGill Integrated Program in Neuroscience (SJ), an Natural Sciences and 457 Engineering Research Council (NSERC) Undergraduate Student Research Award (JS), a Canada 458 Graduate Scholarship -MSc from the Canadian Institutes of Health Research (CIHR) (EF), and 459 CIHR Operating Grant (MOP-130570) and Project Grant (PJT-153150) (AJW).

Supplementary Information

463 Supplementary Figs. 1-8

464 Supplementary Table 1 
a

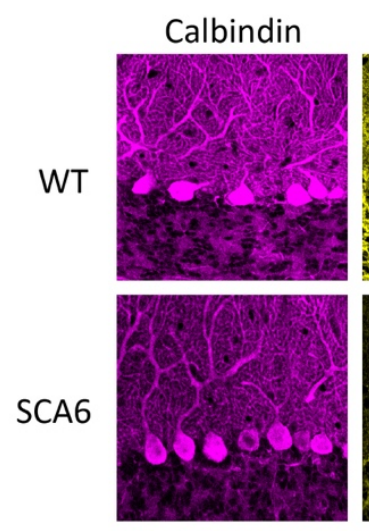

b

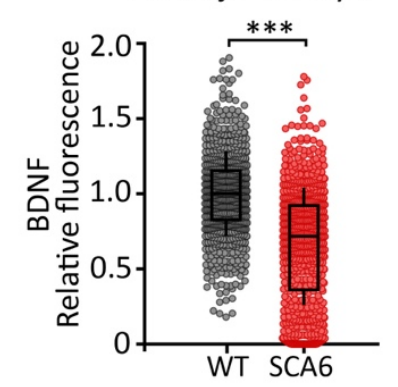

e

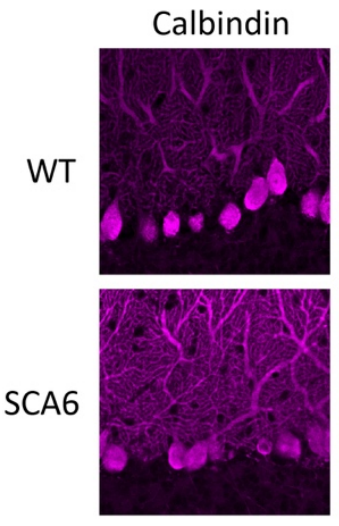

$f$

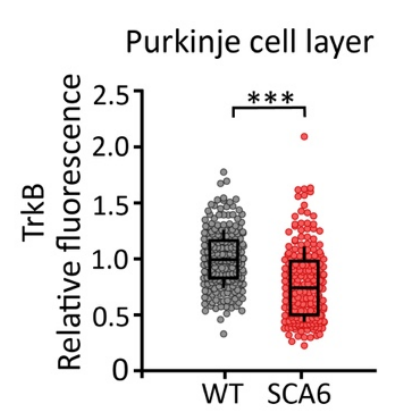

BDNF
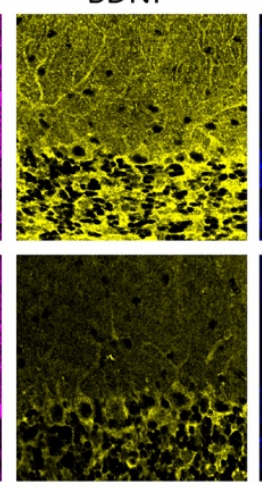

Molecular layer

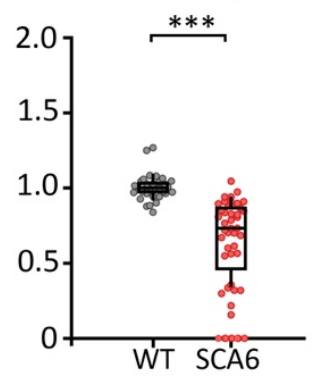

TrkB
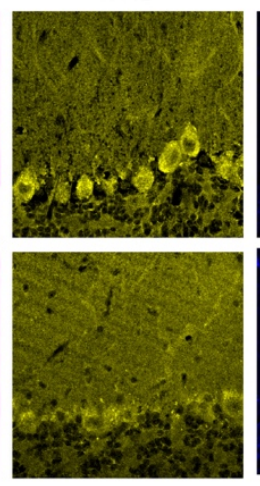

g

Molecular layer

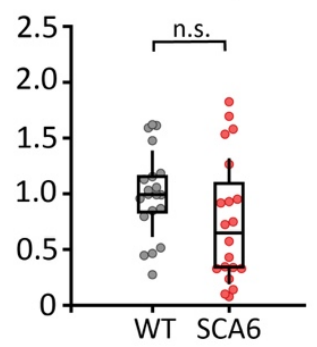

GFAP
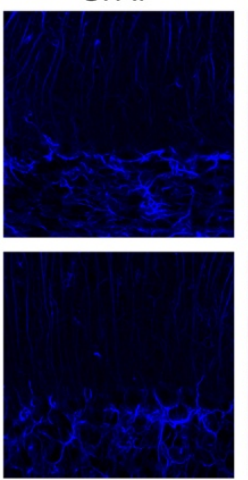

d

Granule cell layer

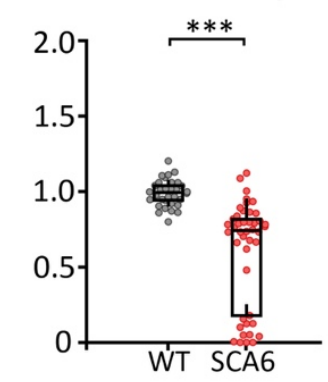

Merge
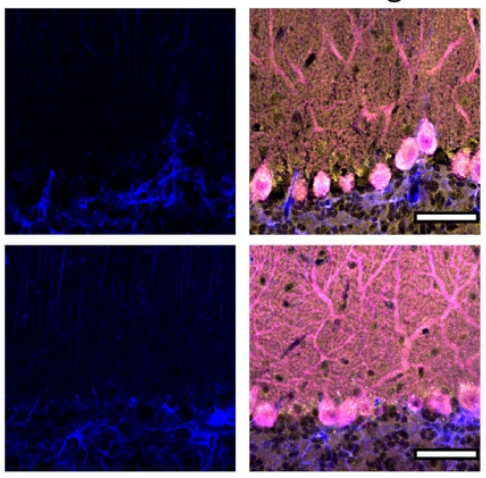

h Granule cell layer

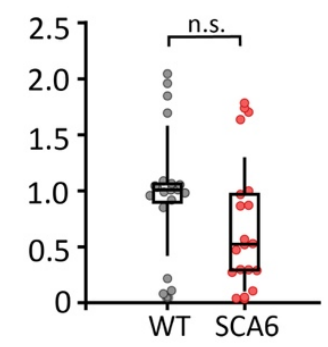


469 (a) Representative images show BDNF is expressed in all three layers of the WT cerebellar cortex 470 at 7 months but expression is decreased in $\mathrm{SCA} 6^{84 \mathrm{Q} / 84 \mathrm{Q}}$ mice. In the upper layers, BDNF 471 colocalizes with Purkinje cells (anti-calbindin) with little labeling in Bergmann glia (anti-GFAP). 472 Scale bar $=50 \mu \mathrm{m}$. (b-d) Quantification showing BDNF levels are reduced in all the three cortical 473 layers: (b) Purkinje cell layer $(\mathrm{P}<0.0001)$, (c) the molecular layer $(\mathrm{P}<0.0001)$, and (d) the granule 474 cell layer $(\mathrm{P}=0.0002)$. 4 regions of interest $(\mathrm{ROI})$ were analyzed for each molecular layer and 475 granule cell measurement per animal; WT N $=5$ mice, $\mathrm{n}=897$ Purkinje cell somata; SCA6 ${ }^{84 \mathrm{Q} / 84 \mathrm{Q}}$ $476 \mathrm{~N}=6$ mice, $\mathrm{n}=912$ Purkinje cell somata. (e) However, TrkB immunoreactivity is reduced only in 477 Purkinje cell somata in SCA6 $6^{84 \mathrm{Q} / 84 \mathrm{Q}}$ mice. Scale bar $=50 \mu \mathrm{m}$. (f-h) Quantification of TrkB 478 expression in the three cortical layers. (f) TrkB is reduced in the Purkinje cell layer, but not (g) the 479 molecular layer (not significantly different, Students $t$ test, $\mathrm{P}=0.12$ ), or $(\mathbf{h})$ the granule cell layer $480(\mathrm{P}=0.12)$. WT $\mathrm{N}=4$ mice, $\mathrm{n}=167$ Purkinje cell somata, $\mathrm{SCA} 6^{84 \mathrm{Q} / 84 \mathrm{Q}} \mathrm{N}=4$ mice, $\mathrm{n}=125$ Purkinje 481 cell somata; Mann Whitney $U$ test unless otherwise indicated (when data were normally 482 distributed). $* * * \mathrm{P}<0.001$, n.s. $\mathrm{P}>0.05$. 
a

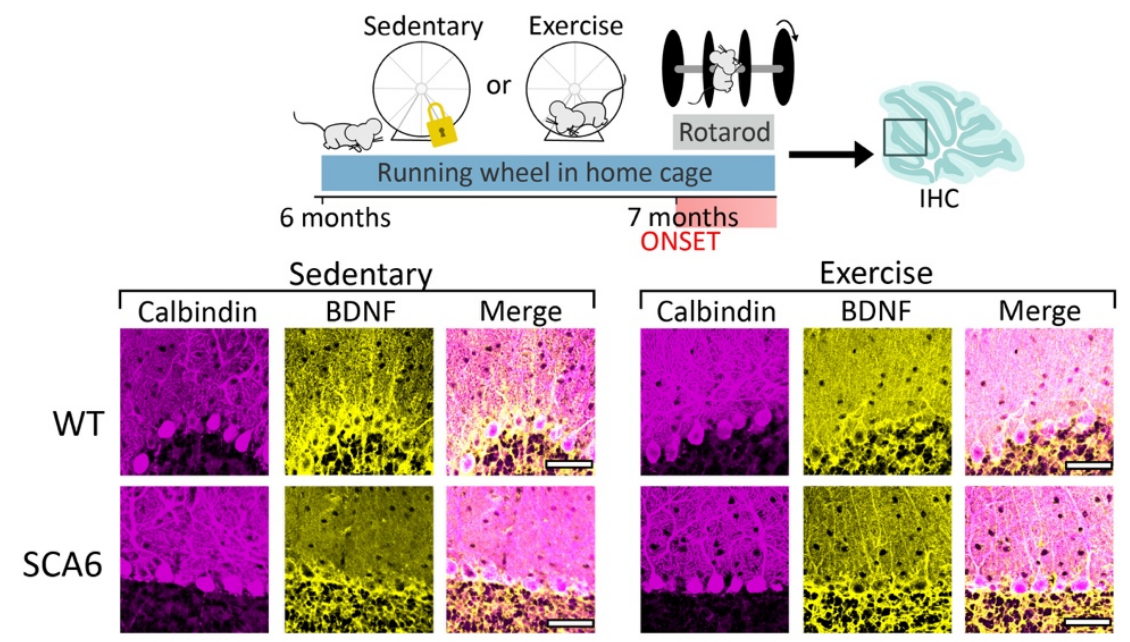

b

C
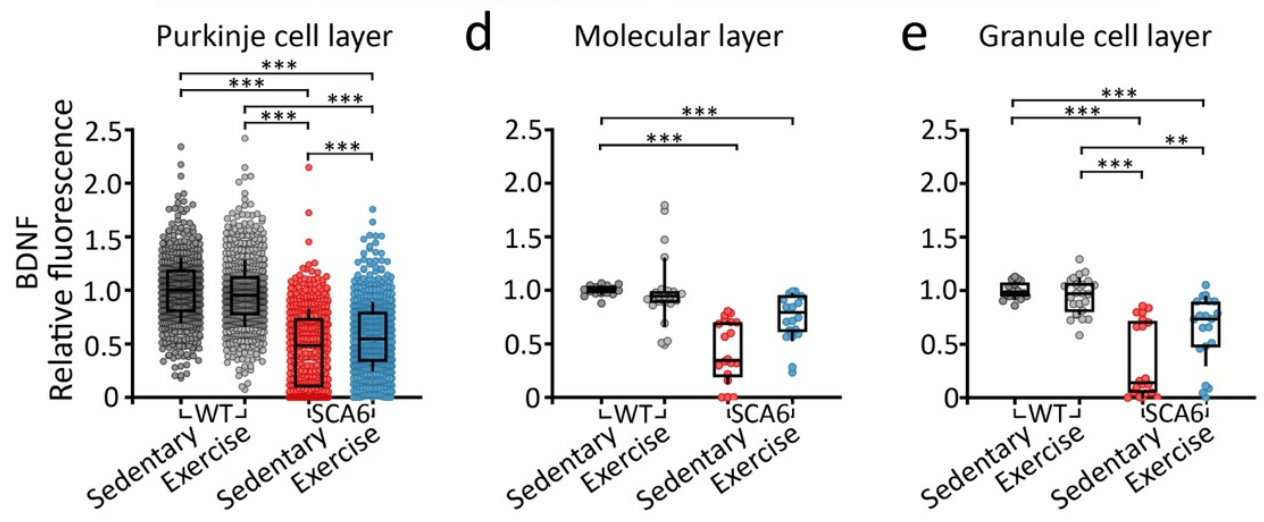

$f$

Sedentary

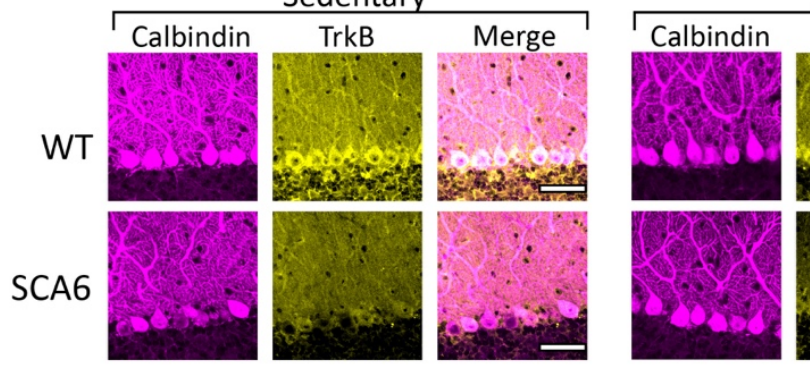

Exercise
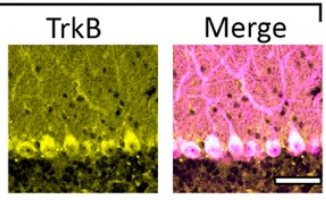

h Molecular layer

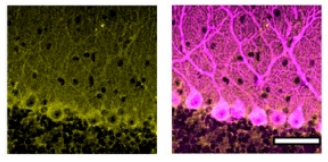

g
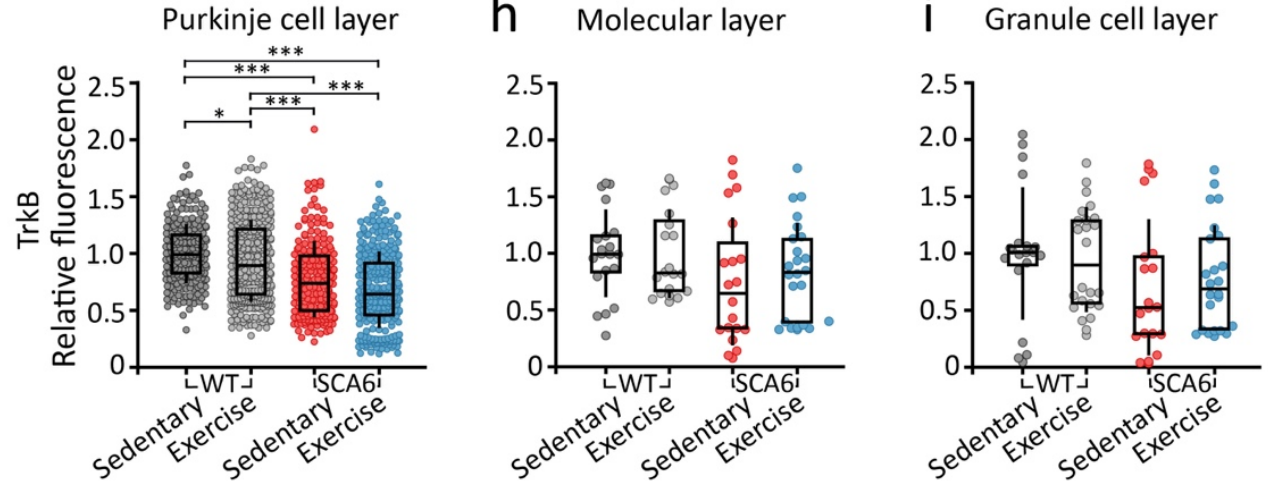

Figure 2: Exercise increases cerebellar BDNF levels in SCA6 ${ }^{84 Q / 84 Q}$ mice. 
487 (a) Schematic of exercise protocol. (b) BDNF immunoreactivity in sedentary (left) and exercise 488 (right) WT (top) and SCA6 ${ }^{84 \mathrm{Q} / 84 \mathrm{Q}}(\mathrm{SCA} 6$, bottom) mice at 7 months. Scale bars $=50 \mu \mathrm{m}$. (c-e) 489 Cerebellar BDNF expression in cortical layers: (WT $\mathrm{N}=3$ mice, WT exercise $\mathrm{N}=3$ mice, SCA6 $490 \mathrm{~N}=3$ mice, SCA6 exercise $\mathrm{N}=4$ mice) (c) Purkinje cell layer (All comparisons $\mathrm{P}<0.0001$ except 491 WT Sedentary vs WT Exercise P $=0.45$ and SCA6 Sedentary vs SCA6 Exercise, P =0.0016); (d) 492 the molecular layer (All comparisons $\mathrm{P}<0.005$ except WT Sedentary vs WT Exercise, $\mathrm{P}=0.41$; 493 WT Sedentary vs SCA6 Exercise, P = 0.022; WT Exercise vs SCA6 Sedentary, P = 0.0063; WT 494 Exercise vs SCA6 Exercise, $\mathrm{P}=0.27$ ); and (e) the granule cell layer (WT Sedentary vs Exercise, $495 \mathrm{P}>0.99$; WT Sedentary vs SCA6 Sedentary, $\mathrm{P}<0.0001$; WT Sedentary vs SCA6 Exercise, $\mathrm{P}=$ 496 0.0008; WT Exercise vs SCA6 Sedentary, P = 0.0004; WT Exercise vs SCA6 Exercise, P =0.0063; 497 SCA6 Sedentary vs SCA6 Exercise, P>0.99). (f) Cerebellar TrkB expression in Sedentary (left) 498 and Exercised (right) WT (top) and SCA6 ${ }^{84 \mathrm{Q} / 84 \mathrm{Q}}$ (bottom) mice at 7 months. Scale bars $=100 \mu \mathrm{m}$. 499 (g-i) Quantification of changes in TrkB expression. (WT $\mathrm{N}=4$ mice, WT exercise $\mathrm{N}=6$ mice, 500 SCA6 $\mathrm{N}=4$ mice, SCA6 exercise $\mathrm{N}=6$ mice) (g) Purkinje cell layer $(\mathrm{P}<0.0001$ for all 501 comparisons except WT Sedentary vs WT Exercise P = 0.016, SCA6 Sedentary vs SCA6 Exercise $502 \mathrm{P}=0.14) ;(\mathbf{h})$ : molecular layer $(\mathrm{P}>0.99$ for all comparisons except WT Sedentary vs SCA6 503 Sedentary, $\mathrm{P}=0.25$; WT Exercise vs SCA6 Sedentary, $\mathrm{P}=0.50)$, and $(\mathbf{i})$ the granule cell layer $(\mathrm{P}$ $504>0.99$ for all comparisons except WT Sedentary vs SCA6 Sedentary, P = 0.31; WT Exercise vs 505 SCA6 Sedentary, $\mathrm{P}=0.30)$. All data is analyzed with one-way ANOVA followed by Dunn's 506 multiple comparisons test. $* \mathrm{P}<0.05, * * \mathrm{P}<0.01, * * * \mathrm{P}<0.005, \mathrm{P}>0.05$ when comparison is 507 not shown. 
a

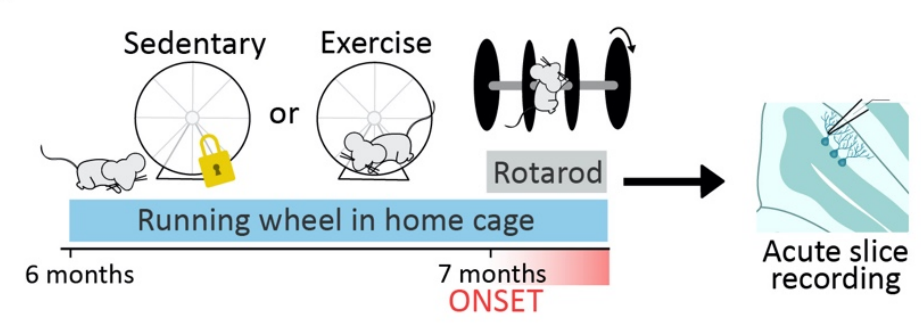

b 200$] \bullet$ WT Sedentary
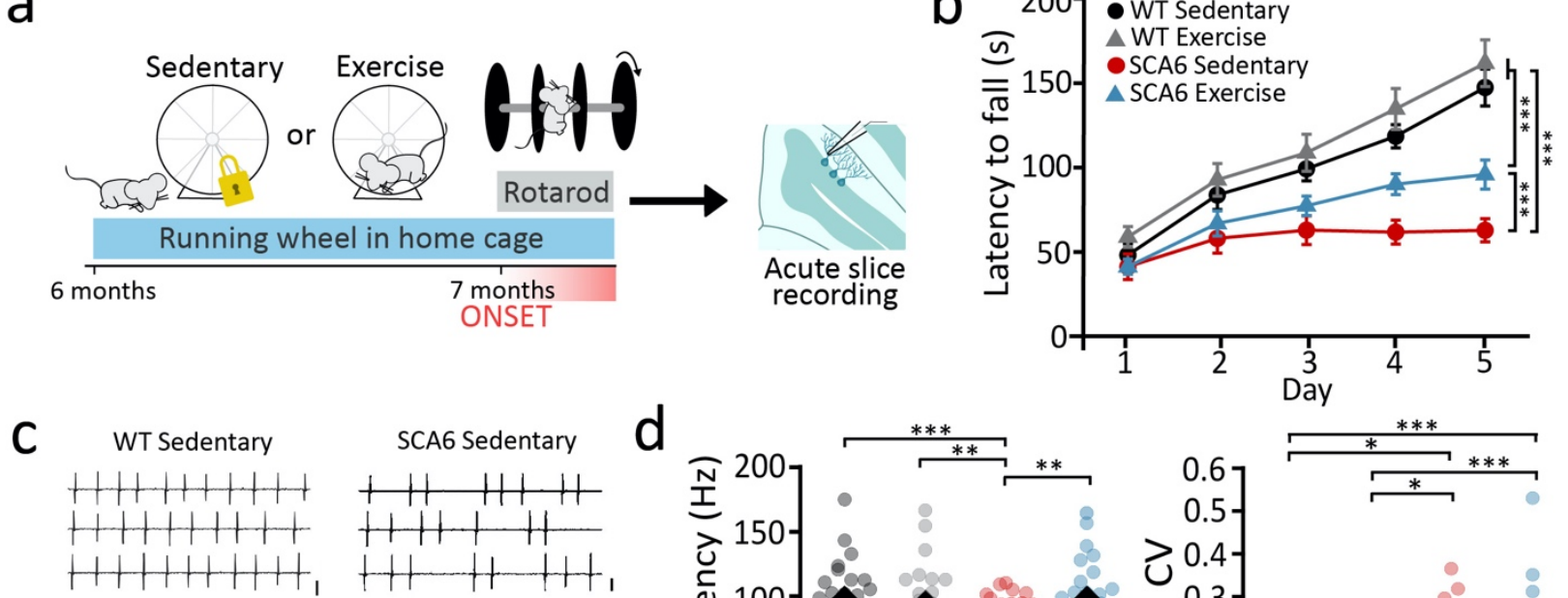

WT Exercise

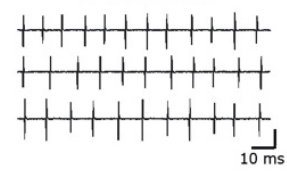

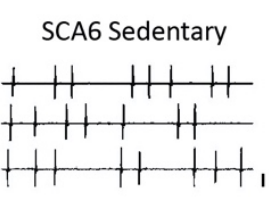

SCA6 Exercise

$\mathrm{HWH}+\mathrm{H}+$

$\mathrm{HHHH}+\mathrm{HH}_{10 \mathrm{~ms}}$
H H H H H
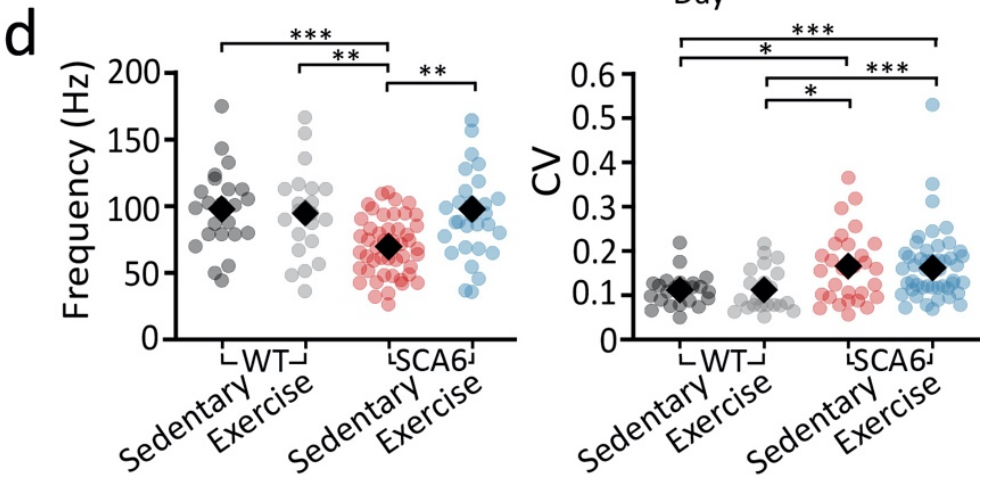

509

510

511

512

513

514

515

516

517

518

519

520

521

522

523

524

525

526

527

Figure 3: Exercise rescues motor coordination and Purkinje cell firing deficits in SCA6 ${ }^{840 / 84 Q}$ mice.

(a) Schematic of exercise protocol. (b) Motor coordination is assessed with an accelerating rotarod assay after 1 month of voluntary wheel running by measuring the latency to fall for 5 days of testing (one-way ANOVA followed by Tukey's multiple comparisons test, $\mathrm{P}<0.0001$ for all comparisons except WT Sedentary vs Exercise, $\mathrm{P}=0.4147$ and SCA6 Sedentary vs Exercise $\mathrm{P}=$ 0.0002; WT Sedentary, N = 10; WT Exercise, N = 13, SCA6 Sedentary, N = 13; SCA6 Exercise, $\mathrm{N}=20$ ). (c) Sample juxtacellular Purkinje cell recordings from WT (left) and SCA6 ${ }^{84 Q / 84 Q}$ (right) Sedentary (top) or exercise (bottom) mouse cerebellar acute slices. (d) Purkinje cell firing frequency (left) is restored in SCA6 ${ }^{84 Q / 84 Q}$ Exercise mice (one-way ANOVA followed by Tukey's multiple comparisons test, WT Sedentary vs WT Exercise, P $=0.98$, WT Sedentary vs SCA6 Sedentary, $\mathrm{P}=0.001$; WT Sedentary vs SCA6 Exercise, $\mathrm{P}=0.90$; WT Exercise vs SCA6 Sedentary, P = 0.0062, WT Exercise vs SCA6 Exercise P > 0.99; SCA6 Sedentary vs Exercise, P $=0.0055$ ). However, action potential regularity (as measured by the coefficient of variation, $\mathrm{CV}$, of interspike intervals) is not restored by Exercise (WT Sedentary vs Exercise, $\mathrm{P}=0.99$; WT Sedentary vs SCA6 Sedentary, P =0.011; WT Sedentary vs SCA6 Exercise, P =0.0026; WT Exercise vs SCA6 Sedentary, $\mathrm{P}=0.014$; WT Exercise vs SCA6 exercise, $\mathrm{P}=0.0036$, SCA6 Sedentary vs Exercise, $\mathrm{P}=0.798) . \mathrm{n}=29$ cells from $\mathrm{N}=3$ mice WT Sedentary mice; $\mathrm{n}=21$ cells 
528 from $\mathrm{N}=3$ mice WT Exercise mice; $\mathrm{n}=47$ cells from $\mathrm{N}=3$ mice SCA6 Sedentary mice; $\mathrm{n}=29$

529 cells from $\mathrm{N}=3$ mice SCA6 Exercise mice. ${ }^{*} \mathrm{P}<0.05,{ }^{* *} \mathrm{P}<0.01,{ }^{* * *} \mathrm{P}<0.005, \mathrm{P}>0.05$ when 530 not shown.

531 

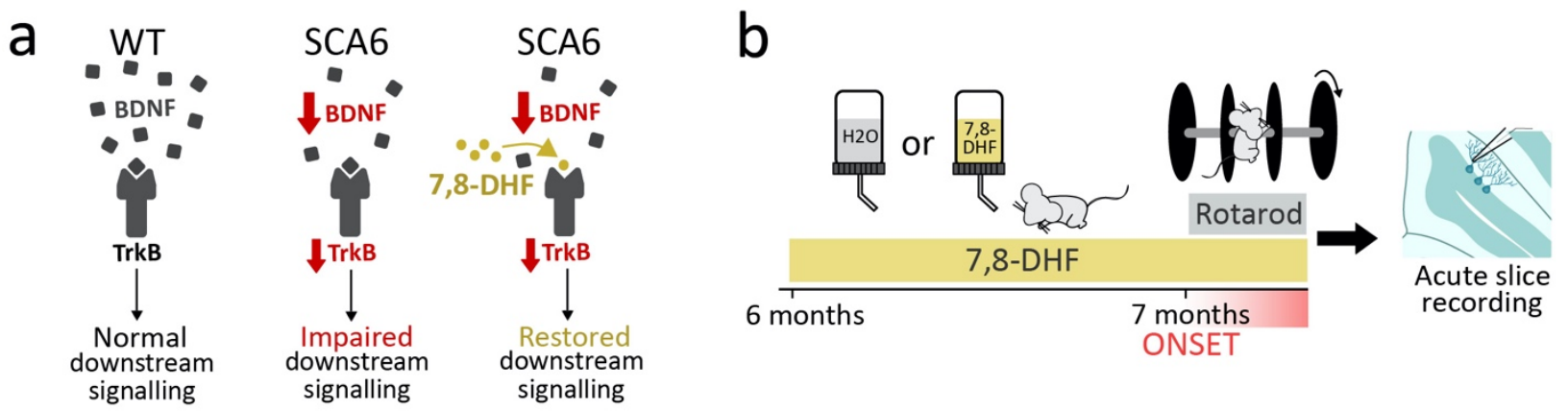

$\mathrm{C}$

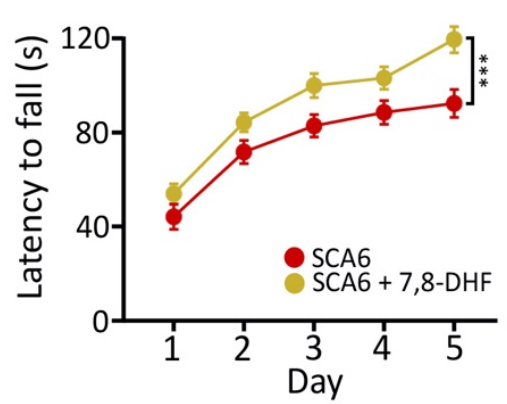

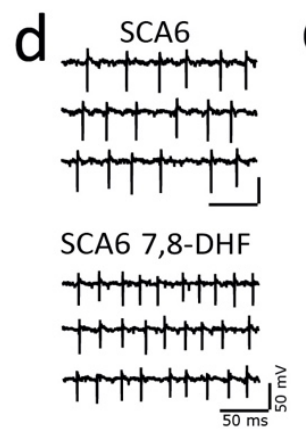

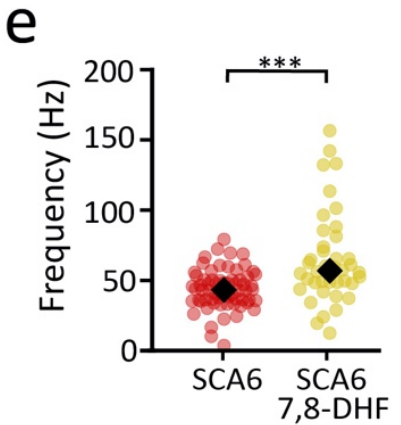

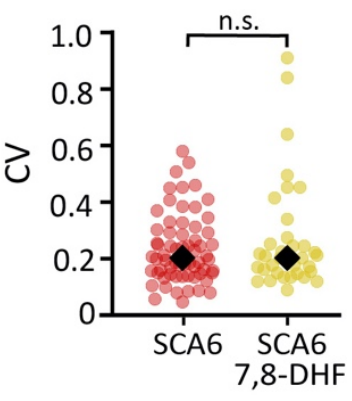

533

534

535

536

537

538

539

540

541

542

543

544

545

Figure 4: The BDNF mimetic 7,8-DHF restores ataxia and Purkinje cell firing deficits in $\mathrm{SCA} 6^{84 \mathrm{Q} / 84 \mathrm{Q}}$ mice at disease onset.

(a) Proposed model of altered BDNF-TrkB signaling in SCA6 $6^{84 \mathrm{Q} / 84 \mathrm{Q}}$ mice and its subsequent restoration with BDNF mimetic 7,8-DHF, a TrkB agonist. (b) Schematic of 7,8-DHF administration protocol. (c) 7,8-DHF improves motor coordination in SCA6 ${ }^{84 \mathrm{Q} / 84 \mathrm{Q}}$ mice with rotarod assay (SCA6 $\mathrm{N}=14$ mice, SCA6 + DHF $\mathrm{N}=14$ mice, significantly different, two-tailed Students $t$-test, $\mathrm{P}=0.0014)$. (d) Example traces from juxtacellular recordings of Purkinje cell firing at 7 months after 1 month of 7,8-DHF administration. (e) Purkinje cell firing frequency is elevated after 7,8-DHF administration $(n=64$ cells from $\mathrm{N}=3$ mice SCA6 mice; $\mathrm{n}=42$ cells from $\mathrm{N}=3$ mice SCA6 + 7,8-DHF, significantly different, Mann Whitney $U$ test, $\mathrm{P}<0.0001$, left). However, Purkinje cell firing regularity is unchanged with 7,8-DHF administration (CV not significantly different, Mann Whitney $U$ test, $\mathrm{P}=0.86$, right). *** $\mathrm{P}<0.005$, n.s. $\mathrm{P}>0.05$. 
a

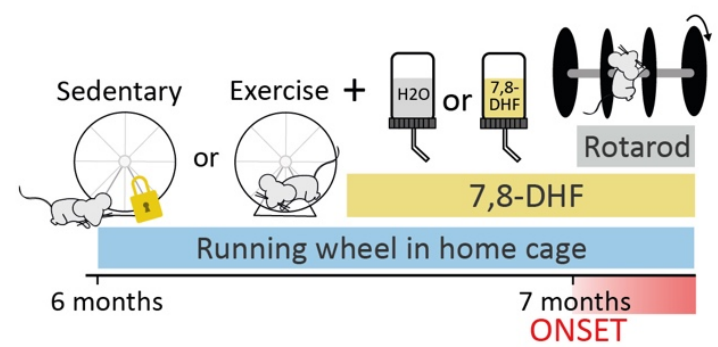

b

If Exercise and 7,8-DHF:

C
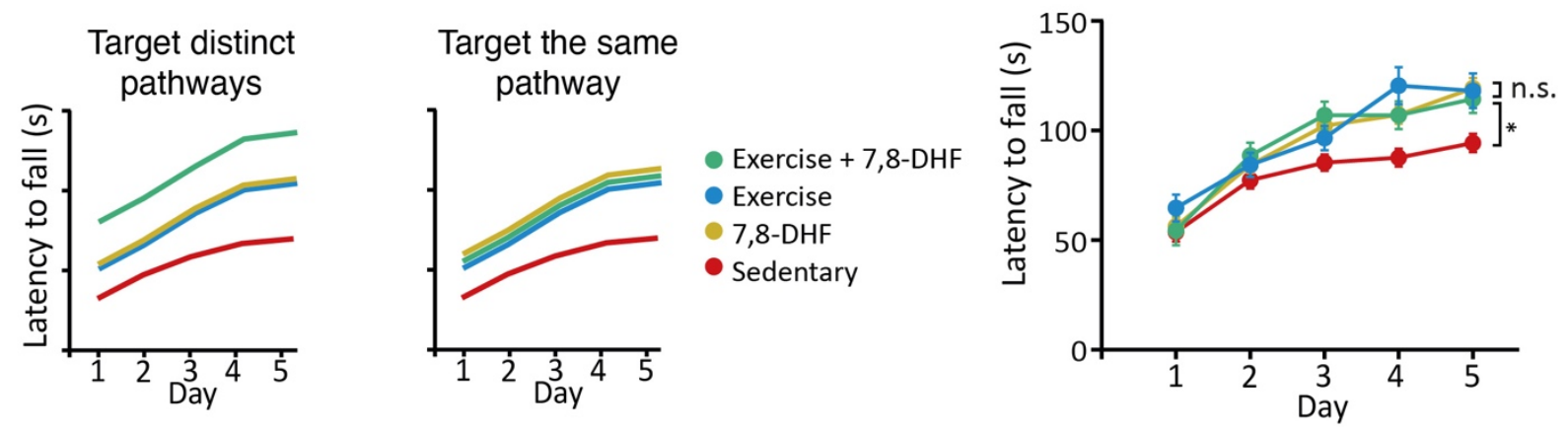

Figure 5: Combining exercise with administration of a TrkB agonist provides no further rescue of ataxia in SCA6 ${ }^{84 Q / 84 Q}$ mice.

(a) Schematic of exercise and 7,8-DHF (DHF) combination therapy on SCA6 ${ }^{84 \mathrm{Q} / 84 \mathrm{Q}}$ mice (SCA6).

(b) Schematics of possible outcomes if exercise and 7,8-DHF are additive suggesting they act via distinct pathways (left), or if no further improvement is observed in motor coordination when exercise and 7,8-DHF are co-administered, suggesting they act on same signaling pathway (right). (c) No additional improvement is observed when exercise and 7,8-DHF administration are combined, and exercise and 7,8-DHF improve motor coordination similarly suggesting they act on the same pathway. (one-way ANOVA followed by Tukey's multiple comparison test; SCA6

556 Sedentary vs SCA6 + DHF, P = 0.0009; SCA6 Sedentary vs SCA6 Exercise, P = 0.024; SCA6

557 Sedentary vs SCA6 Exercise + DHF, P =0.045; SCA6 + DHF vs SCA6 Exercise + DHF, P=0.93;

558 SCA6 Exercise vs SCA6 Exercise + DHF, P = 0.98; SCA6 + DHF vs SCA6 Exercise, P > 0.99;

559 SCA6 Sedentary $N=19$ mice, SCA6 Exercise $N=12$ mice, SCA6 + DHF N = 15 mice, SCA6 Exercise + DHF $\mathrm{N}=9$ mice). $* \mathrm{P}<0.05$, n.s. $\mathrm{P}>0.05$. 


\section{a}

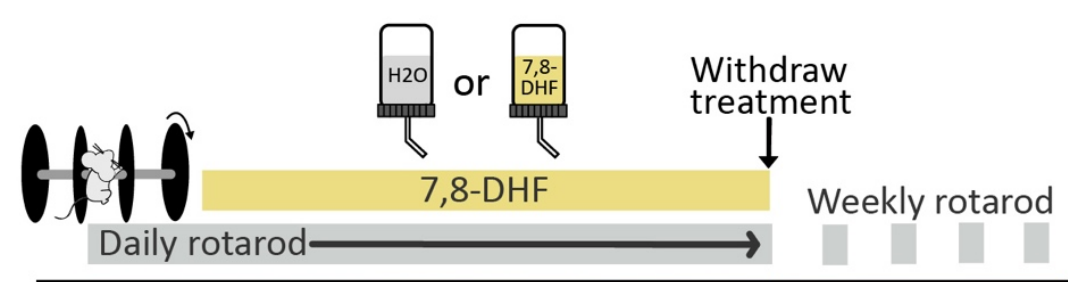

7 months ONSET
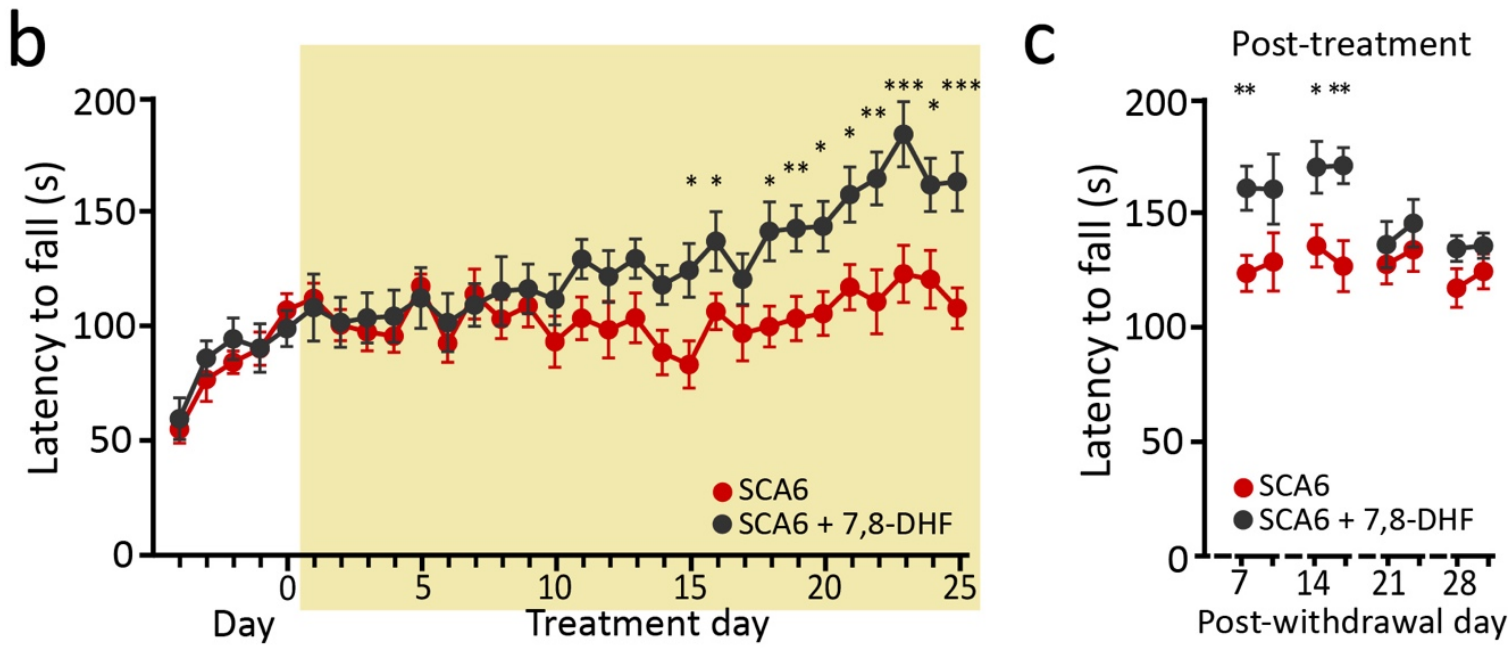

562

563

564

565

566

567

568

569

570

571

572

573

574

575

Figure 6: Chronic administration of 7,8-DHF leads to a slow timecourse of improvement of ataxia in $\mathrm{SCA} 6^{84 Q / 84 Q}$ mice

(a) Schematic of 7,8-DHF administration protocol. (b) SCA6 ${ }^{84 \mathrm{Q} / 84 \mathrm{Q}}$ mice (SCA6) were administered 7,8-DHF for 25 days after 5 days of baseline. Improvement in motor coordination was observed after $\sim 2$ weeks (SCA6 $\mathrm{N}=4$ mice, SCA6 $+7,8$-DHF $\mathrm{N}=4$ mice, all days compared with Mann Whitney $U$ tests or Student's $t$ tests, days marked with asterisks were significantly different; treatment day $14, \mathrm{P}=0.030$; day $15, \mathrm{P}=0.013$; day $18, \mathrm{P}=0.011$; day $19, \mathrm{P}=0.0081$; day $20, \mathrm{P}=0.013$; day $21, \mathrm{P}=0.014$; day $22, \mathrm{P}=0.0061$; day $23, \mathrm{P}=0.0031$; day $24, \mathrm{P}=0.023$; day $25, \mathrm{P}=0.0020$, all other $\mathrm{P}$ values were $>0.05$ ). (c) Motor performance remained elevated for $\sim 2$ weeks after 7,8-DHF withdrawal (SCA6 $\mathrm{N}=3$, SCA6 DHF (withdrawn) $\mathrm{N}=3$, all days compared with Mann Whitney $U$ tests or Student's $t$ tests, days marked with asterisks were significantly different: day $7, \mathrm{P}=0.0092$; day $14, \mathrm{P}=0.011$; day $15, \mathrm{P}=0.0062$, all other $\mathrm{p}$ values were $>0.05)$. $* \mathrm{P}<0.05, * * \mathrm{P}<0.01, \mathrm{P}>0.05$ when not shown. 
a

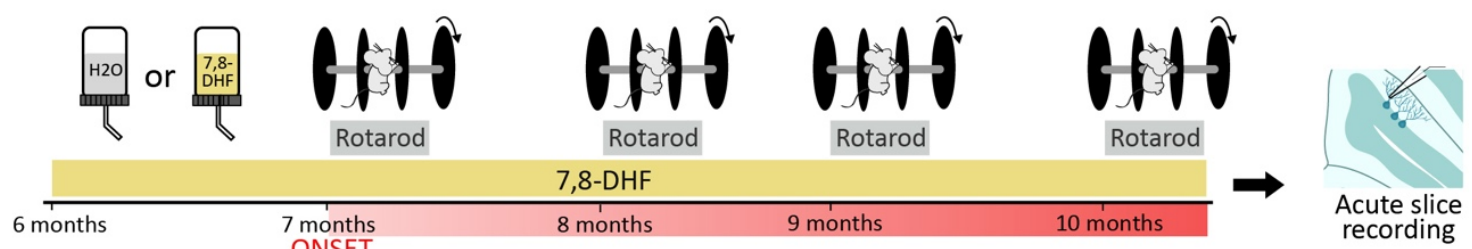

b

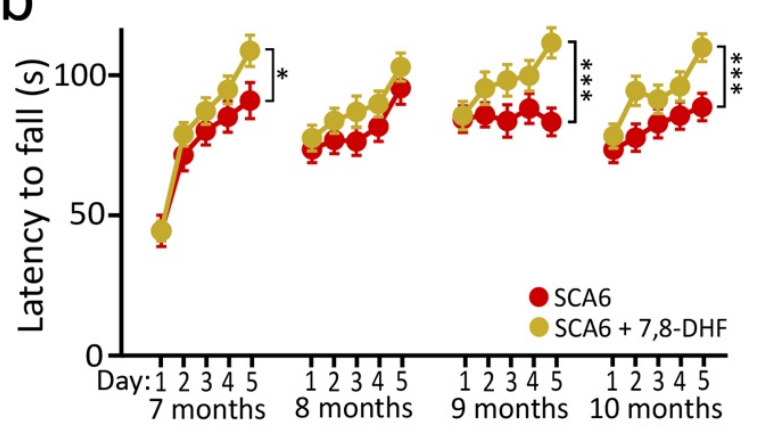

C
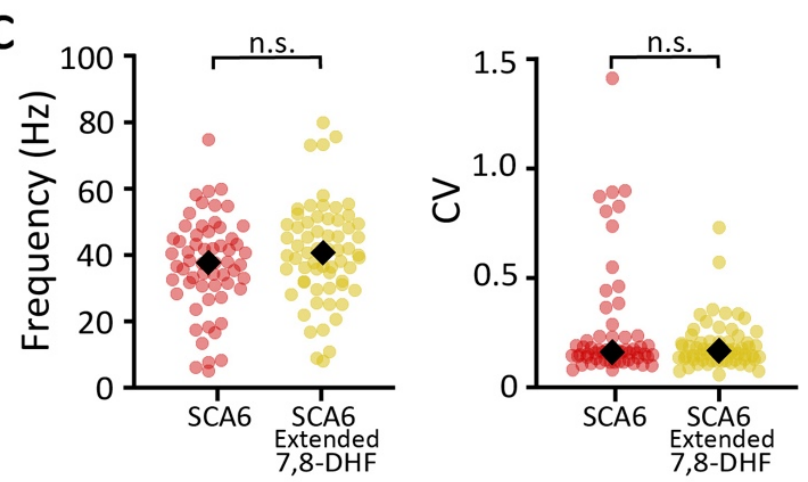

d

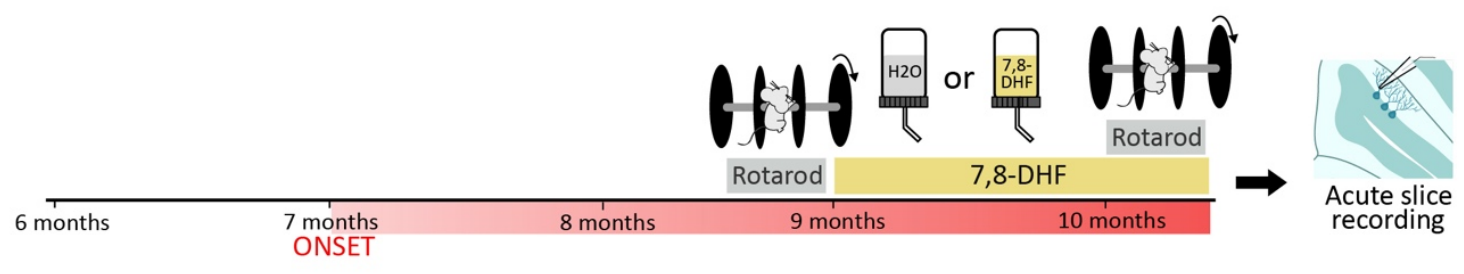

e

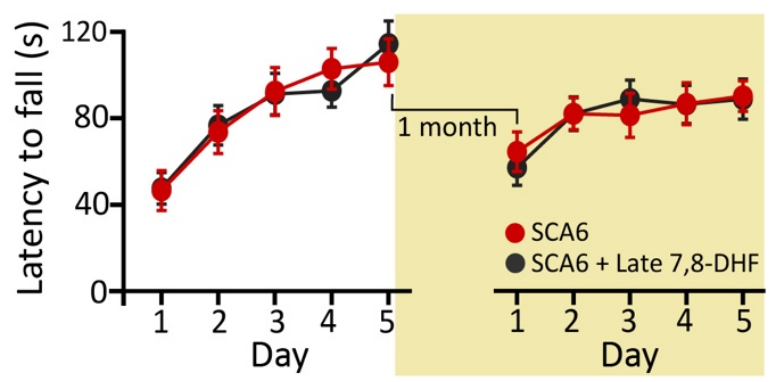

$f$

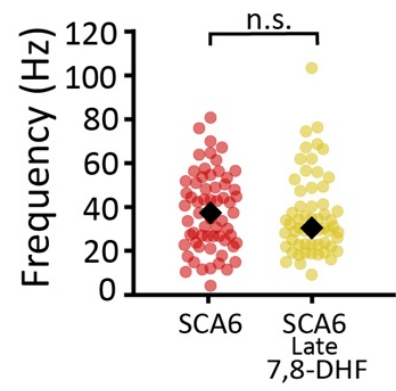

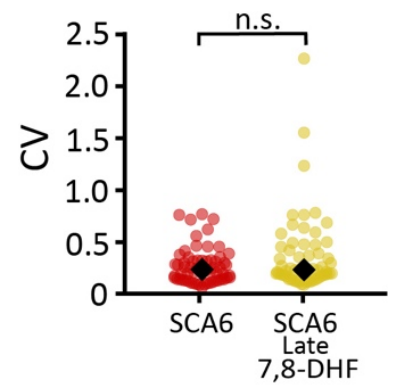

576

577

578

579

580

581

582

583

584

585

Figure 7: Early, but not late, 7,8-DHF administration rescues deficits as disease progresses in SCA6 ${ }^{84 Q / 84 Q}$ mice

(a) Schematic of long-term chronic 7,8-DHF (DHF) administration in SCA6 ${ }^{84 Q / 84 Q}$ mice started near disease onset. (b) Mice treated chronically with 7,8-DHF performed better at 7, 9, and 10 months old (SCA6 $\mathrm{N}=11$ mice, SCA6 DHF N $=9$ mice, all months were compared using T-test, 7 month $\mathrm{p}=0.041,8$ month $\mathrm{p}=0.33,9$ month $\mathrm{p}<0.0001,10$ month $\mathrm{p}=0.00014)$ (c) Purkinje cell firing rate (left) was no longer restored after 4 months of drug administration (SCA6, $\mathrm{n}=58$ cells from $\mathrm{N}=2$ mice; SCA6 DHF $\mathrm{n}=62$ cells from $\mathrm{N}=3$ mice; not significantly different, two-tailed Student's t test, $\mathrm{P}=0.22$ ), nor was regularly affected (not significantly different, Mann-Whitney 
$586 U$ test, $\mathrm{P}=0.63$ ). (d) Schematic of 7,8-DHF administration started in late disease progression. (e)

587 Late-administered 7,8-DHF does not restore firing deficits at 10 months when treatment starts after

588 disease onset at 9 months. (not significantly different before or after DHF administration, two-

589 tailed Student's $t$ tests; before $\mathrm{P}=0.91$, after $\mathrm{P}=0.58$; SCA6 $\mathrm{N}=5$ mice; SCA6 late DHF $\mathrm{N}=4$

590 mice). (f) Purkinje cell firing rate and regularity (CV) are not significantly different after late

591 administration of 7,8-DHF (left: frequency not significantly different, Mann Whitney $U$ test, $\mathrm{P}=$

592 0.46; right, $\mathrm{CV}$ not significantly different, $\mathrm{P}=0.17$; $\mathrm{SCA} 6, \mathrm{n}=62$ cells from $\mathrm{N}=3$ mice, SCA6

593 late $\mathrm{DHF}, \mathrm{n}=59$ cells from $\mathrm{N}=3$ mice). $* \mathrm{P}<0.05, * * * \mathrm{P}<0.001$, n.s. $\mathrm{P}>0.05$. 
597

598

599

600

601

602

603

604

605

606

607

608

609

610

611

612

613

614

615

616

617

618

619

620

621

622

\section{References}

1. Zhuchenko, O. et al. Autosomal dominant cerebellar ataxia (SCA6) associated with small polyglutamine expansions in the alpha 1A-voltage-dependent calcium channel. Nat.

Genet. 15, 62-9 (1997).

2. Solodkin, A. \& Gomez, C. M. Spinocerebellar ataxia type 6. Handb. Clin. Neurol. 103, $461-473$ (2012).

3. Watase, K. et al. Spinocerebellar ataxia type 6 knockin mice develop a progressive neuronal dysfunction with age-dependent accumulation of mutant CaV2.1 channels. Proc. Natl. Acad. Sci. U. S. A. 105, 11987-92 (2008).

4. Jayabal, S. et al. Rapid Onset of Motor Deficits in a Mouse Model of Spinocerebellar Ataxia Type 6 Precedes Late Cerebellar Degeneration. eNeuro 2, ENEURO.0094-15.2015 (2015).

5. Zuccato, C. \& Cattaneo, E. Brain-derived neurotrophic factor in neurodegenerative diseases. Nature Reviews Neurology vol. 5 311-322 (2009).

6. Takahashi, M. et al. Reduced brain-derived neurotrophic factor (BDNF) mRNA expression and presence of BDNF-immunoreactive granules in the spinocerebellar ataxia type 6 (SCA6) cerebellum. Neuropathology 32, 595-603 (2012).

7. Orr, H. T. \& Zoghbi, H. Y. Trinucleotide repeat disorders. Annu. Rev. Neurosci. 30, 575$621(2007)$.

8. Murer, M. G., Yan, Q. \& Raisman-Vozari, R. Brain-derived neurotrophic factor in the control human brain, and in Alzheimer's disease and Parkinson's disease. Progress in Neurobiology vol. 63 71-124 (2001).

9. Phillips, H. S. et al. BDNF mRNA is decreased in the hippocampus of individuals with Alzheimer's disease. Neuron 7, 695-702 (1991).

10. Hourez, R. et al. Aminopyridines Correct Early Dysfunction and Delay Neurodegeneration in a Mouse Model of Spinocerebellar Ataxia Type 1. J. Neurosci. 31, 
11795-11807 (2011).

624 11. Mellesmoen, A., Sheeler, C., Ferro, A., Rainwater, O. \& Cvetanovic, M. Brain Derived 625 Neurotrophic Factor (BDNF) Delays Onset of Pathogenesis in Transgenic Mouse Model of Spinocerebellar Ataxia Type 1 (SCA1). Front. Cell. Neurosci. 12, 509 (2019).

627 12. Meng, H., Larson, S. K., Gao, R. \& Qiao, X. BDNF transgene improves ataxic and motor 628 behaviors in stargazer mice. Brain Res. 1160, 47-57 (2007).

629

630

631

632

633

13. Salomova, M., Tichanek, F., Jelinkova, D. \& Cendelin, J. Abnormalities in the cerebellar levels of trophic factors BDNF and GDNF in pcd and lurcher cerebellar mutant mice. Neurosci. Lett. 725, 134870 (2020).

14. Parrini, M. et al. Aerobic exercise and a BDNF-mimetic therapy rescue learning and memory in a mouse model of Down syndrome. Sci. Rep. 7, 16825 (2017).

15. Neeper, S. A., Gómez-Pinilla, F., Choi, J. \& Cotman, C. W. Physical activity increases mRNA for brain-derived neurotrophic factor and nerve growth factor in rat brain. Brain Res. 726, 49-56 (1996).

16. Neeper, S. A., Góauctemez-Pinilla, F., Choi, J. \& Cotman, C. Exercise and brain neurotrophins. Nature vol. 373109 (1995).

17. Gold, S. M. et al. Basal serum levels and reactivity of nerve growth factor and brainderived neurotrophic factor to standardized acute exercise in multiple sclerosis and controls. J. Neuroimmunol. 138, 99-105 (2003).

18. Rojas Vega, S. et al. Acute BDNF and cortisol response to low intensity exercise and following ramp incremental exercise to exhaustion in humans. Brain Res. 1121, 59-65 (2006).

19. Lourenco, M. V. et al. Exercise-linked FNDC5/irisin rescues synaptic plasticity and memory defects in Alzheimer's models. Nat. Med. 25, 165-175 (2019).

20. Choi, S. H. et al. Combined adult neurogenesis and BDNF mimic exercise effects on cognition in an Alzheimer's mouse model. Science 361, eaan8821 (2018).

21. Jayabal, S., Chang, H. H. V., Cullen, K. E. \& Watt, A. J. 4-aminopyridine reverses ataxia 
650

651

652

653

654

655

656

657

658

659

660

661

662

663

664

665

666

667

668

669

670

671

672

673

674

675

676

and cerebellar firing deficiency in a mouse model of spinocerebellar ataxia type 6. Sci. Rep. (2016) doi:10.1038/srep29489.

22. Cook, A. A., Fields, E. \& Watt, A. J. Losing the beat: contribution of Purkinje cell firing dysfunction to disease, and its reversal. Neuroscience (2020) doi:10.1016/j.neuroscience.2020.06.008.

23. Kordasiewicz, H. B. \& Gomez, C. M. Molecular Pathogenesis of Spinocerebellar Ataxia Type 6. Neurotherapeutics 4, 285-294 (2007).

24. Cechetti, F. et al. Effect of a neuroprotective exercise protocol on oxidative state and BDNF levels in the rat hippocampus. Brain Res. 1188, 182-188 (2008).

25. Inoue, T. et al. Effects of long-term exercise and low-level inhibition of GABAergic synapses on motor control and the expression of BDNF in the motor related cortex. Neurol. Res. 40, 18-25 (2018).

26. Johnson, R. A., Rhodes, J. S., Jeffrey, S. L., Garland, T. \& Mitchell, G. S. HIPPOCAMPAL BRAIN-DERIVED NEUROTROPHIC FACTOR BUT NOT NEUROTROPHIN-3 INCREASES MORE IN MICE SELECTED FOR INCREASED VOLUNTARY WHEEL RUNNING. (2003) doi:10.1016/S0306-4522(03)00422-6.

27. Cheng, P. L. et al. Self-amplifying autocrine actions of BDNF in axon development. Proc. Natl. Acad. Sci. U. S. A. 108, 18430-18435 (2011).

28. Fryer, J. D. et al. Exercise and genetic rescue of SCA1 via the transcriptional repressor Capicua. Science 334, 690-3 (2011).

29. Chuang, C. et al. Treadmill training increases the motor activity and neuron survival of the cerebellum in a mouse model of spinocerebellar ataxia type 1. Kaohsiung J. Med. Sci. 35, 679-685 (2019).

30. Alvarez-Saavedra, M. et al. Voluntary Running Triggers VGF-Mediated Oligodendrogenesis to Prolong the Lifespan of Snf2h-Null Ataxic Mice. Cell Rep. (2016) doi:10.1016/j.celrep.2016.09.030.

31. Ady, V. et al. Altered synaptic and firing properties of cerebellar Purkinje cells in a mouse 
model of ARSACS. J. Physiol. (2018) doi:10.1113/JP275902.

678

679

680

681

682

683

684

685

686

687

688

689

690

691

692

693

694

695

696

697

698

699

700

701

702

703

32. Shakkottai, V. G. et al. Early changes in cerebellar physiology accompany motor dysfunction in the polyglutamine disease spinocerebellar ataxia type 3. J. Neurosci. 31, 13002-14 (2011).

33. Stoyas, C. A. et al. Nicotinamide Pathway-Dependent Sirt1 Activation Restores Calcium Homeostasis to Achieve Neuroprotection in Spinocerebellar Ataxia Type 7. Neuron 105, 630-644.e9 (2020).

34. Liu, C., Chan, C. B. \& Ye, K. 7,8-dihydroxyflavone, a small molecular TrkB agonist, is useful for treating various BDNF-implicated human disorders. Transl. Neurodegener. 5, 2 (2016).

35. Paulsen, J. S. et al. A review of quality of life after predictive testing for and earlier identification of neurodegenerative diseases. Progress in Neurobiology vol. 110 2-28 (2013).

36. Miyai, I. et al. Cerebellar ataxia rehabilitation trial in degenerative cerebellar diseases. Neurorehabil. Neural Repair 26, 515-22 (2012).

37. Ilg, W. et al. Intensive coordinative training improves motor performance in degenerative cerebellar disease. Neurology (2009) doi:10.1212/WNL.0b013e3181c33adf.

38. Chang, Y. J. et al. Cycling regimen induces spinal circuitry plasticity and improves leg muscle coordination in individuals with spinocerebellar ataxia. Arch. Phys. Med. Rehabil. (2015) doi:10.1016/j.apmr.2015.01.021.

39. Barbuto, S. et al. Phase I randomized single-blinded controlled study investigating the potential benefit of aerobic exercise in degenerative cerebellar disease. Clin. Rehabil. 34, 584-594 (2020).

40. Reycraft, J. T. et al. Exercise Intensity and Recovery on Circulating Brain-derived Neurotrophic Factor. Med. Sci. Sports Exerc. 52, 1210-1217 (2020).

41. Jiménez-Maldonado, A., Rentería, I., García-Suárez, P. C., Moncada-Jiménez, J. \& FreireRoyes, L. F. The impact of high-intensity interval training on brain derived neurotrophic 
factor in brain: A mini-review. Frontiers in Neuroscience vol. 12839 (2018).

705

706

707

708

709

710

711

712

713

714

715

716

717

718

719

720

721

722

723

724

725

726

727

729 730

42. Oliff, H. S., Berchtold, N. C., Isackson, P. \& Cotman, C. W. Exercise-induced regulation of brain-derived neurotrophic factor (BDNF) transcripts in the rat hippocampus. Mol. Brain Res. 61, 147-153 (1998).

43. Klintsova, A. Y., Dickson, E., Yoshida, R. \& Greenough, W. T. Altered expression of BDNF and its high-affinity receptor TrkB in response to complex motor learning and moderate exercise. Brain Res. 1028, 92-104 (2004).

44. Rogers, J. et al. Paradoxical effects of exercise on hippocampal plasticity and cognition in mice with a heterozygous null mutation in the serotonin transporter gene. $\mathrm{Br} . J$. Pharmacol. 176, bph.14760 (2019).

45. Adlard, P. A., Perreau, V. M. \& Cotman, C. W. The exercise-induced expression of BDNF within the hippocampus varies across life-span. Neurobiol. Aging 26, 511-520 (2005).

46. Sheeler, C. et al. Post-symptomatic Delivery of Brain-Derived Neurotrophic Factor (BDNF) Ameliorates Spinocerebellar Ataxia Type 1 (SCA1) Pathogenesis. Cerebellum 110 (2021) doi:10.1007/s12311-020-01226-3.

47. Cunha, C. et al. Brain-derived neurotrophic factor (BDNF) overexpression in the forebrain results in learning and memory impairments. Neurobiol. Dis. 33, 358-368 (2009).

48. Croll, S. D. et al. Brain-derived neurotrophic factor transgenic mice exhibit passive avoidance deficits, increased seizure severity and in vitro hyperexcitability in the hippocampus and entorhinal cortex. Neuroscience 93, 1491-1506 (1999).

49. Papaleo, F. et al. Working memory deficits, increased anxiety-like traits, and seizure susceptibility in BDNF overexpressing mice. Learn. Mem. 18, 534-544 (2011).

50. Schindelin, J. et al. Fiji: An open-source platform for biological-image analysis. Nature Methods vol. 9 676-682 (2012).

51. Rueden, C. T. et al. ImageJ2: ImageJ for the next generation of scientific image data. BMC Bioinformatics 18, 529 (2017). 
bioRxiv preprint doi: https://doi.org/10.1101/2021.02.21.432180; this version posted February 22, 2021. The copyright holder for this preprint (which was not certified by peer review) is the author/funder, who has granted bioRxiv a license to display the preprint in perpetuity. It is made available under aCC-BY-NC-ND 4.0 International license.

731 